\title{
A massive nebula around the luminous blue variable star RMC 143 revealed by ALMA ${ }^{\star, \star \star}$
}

C. Agliozzo ${ }^{1,2,3}$, A. Mehner ${ }^{1}$, N. M. Phillips ${ }^{2}$, P. Leto ${ }^{4}$, J. H. Groh ${ }^{5}$, A. Noriega-Crespo ${ }^{6}$, C. Buemi ${ }^{4}$, F. Cavallaro ${ }^{4}$,

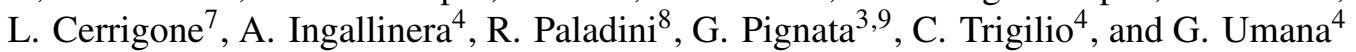

${ }^{1}$ European Southern Observatory, Alonso de Cordova 3107, Vitacura, Santiago de Chile, Chile e-mail: claudia.agliozzo@eso.org

2 European Southern Observatory, Karl-Schwarzschild-Strasse 2, Garching bei München 85748, Germany

3 Departamento de Ciencias Fisicas, Universidad Andres Bello, Avda. Republica 252, Santiago 8320000, Chile

${ }^{4}$ INAF-Osservatorio Astrofisico di Catania, Via S. Sofia 78, 95123 Catania, Italy

5 Trinity College Dublin, The University of Dublin, College Green, Dublin, Ireland

6 Space Telescope Science Institute, 3700 San Martin Dr., Baltimore, MD 21218, USA

7 Joint ALMA Observatory, Alonso de Córdova 3107, Vitacura, Santiago, Chile

${ }^{8}$ Infrared Processing Analysis Center, California Institute of Technology, 770 South Wilson Ave., Pasadena, CA 91125, USA

9 Millennium Institute of Astrophysics (MAS), Nuncio Monseñor Sótero Sanz 100, Providencia, Santiago, Chile

Received 9 February 2019 / Accepted 9 May 2019

\begin{abstract}
The luminous blue variable (LBV) RMC 143 is located in the outskirts of the 30 Doradus complex, a region rich with interstellar material and hot luminous stars. We report the $3 \sigma$ sub-millimetre detection of its circumstellar nebula with ALMA. The observed morphology in the sub-millimetre is different than previously observed with HST and ATCA in the optical and centimetre wavelength regimes. The spectral energy distribution (SED) of RMC 143 suggests that two emission mechanisms contribute to the sub-mm emission: optically thin bremsstrahlung and dust. Both the extinction map and the SED are consistent with a dusty massive nebula with a dust mass of $0.055 \pm 0.018 M_{\odot}$ (assuming $\kappa_{850}=1.7 \mathrm{~cm}^{2} \mathrm{~g}^{-1}$ ). To date, RMC 143 has the most dusty LBV nebula observed in the Magellanic Clouds. We have also re-examined the LBV classification of RMC 143 based on VLT/X-shooter spectra obtained in 2015/16 and a review of the publication record. The radiative transfer code CMFGEN is used to derive its fundamental stellar parameters. We find an effective temperature of $\sim 8500 \mathrm{~K}$, luminosity of $\log \left(L / L_{\odot}\right)=5.32$, and a relatively high mass-loss rate of $1.0 \times 10^{-5} M_{\odot} \mathrm{yr}^{-1}$. The luminosity is much lower than previously thought, which implies that the current stellar mass of $\sim 8 M_{\odot}$ is comparable to its nebular mass of $\sim 5.5 M_{\odot}$ (from an assumed gas-to-dust ratio of 100), suggesting that the star has lost a large fraction of its initial mass in past LBV eruptions or binary interactions. While the star may have been hotter in the past, it is currently not hot enough to ionize its circumstellar nebula. We propose that the nebula is ionized externally by the hot stars in the 30 Doradus star-forming region.
\end{abstract}

Key words. stars: massive - stars: variables: S Doradus - stars: evolution - stars: individual: RMC143 - stars: mass-loss dust, extinction

\section{Introduction}

Luminous blue variables (LBVs), also known as S Doradus variables, are evolved massive stars that exhibit instabilities that are not yet understood (Conti 1984, 1997; Humphreys \& Davidson 1994; Nota \& Lamers 1997, and references therein). The LBV phenomenon is observed at luminosities above $\log \left(L / L_{\odot}\right) \sim 5.2$, corresponding to stars with initial masses of $\gtrsim 20 M_{\odot}$. LBVs experience outbursts with enhanced mass loss during which they appear to make transitions in the Hertzsprung-Russell (HR) diagram from their quiescent hot state $\left(T_{\text {eff }} \sim 12000-30000 \mathrm{~K}\right)$ to lower temperatures $\left(T_{\mathrm{eff}} \sim 8000 \mathrm{~K}\right)$. Outbursts with visual magnitude variations of $1-2 \mathrm{mag}$ are referred to as classical LBV outbursts. During giant eruptions the visual magnitude increases

\footnotetext{
* The ALMA and ATCA maps, and Tables 1 and 4, are only available at the CDS via anonymous ftp to cdsarc.u-strasbg.fr (130.79.128.5) or via http://cdsarc.u-strasbg.fr/viz-bin/ qcat?]/A+A/626/A126

$\star \star$ Phase $3 \mathrm{X}$-shooter spectra are available in the ESO data archive.
}

by more than 2 mag. Examples of stars that have experienced a giant eruption in our Galaxy are P Cygni in the 17th century (e.g. de Groot 1988; Lamers \& de Groot 1992) and $\eta$ Car in the 1840 s (reaching $M_{V} \sim-13$, e.g. Davidson \& Humphreys 1997, 2012; Smith \& Frew 2011).

LBVs are considered to be stars in transition to the WolfRayet (WR) stage (e.g. Langer et al. 1994; Groh et al. 2014, and references therein). However, recent observational and theoretical work suggests that some LBVs could be the immediate progenitors of supernovae (e.g. Kotak \& Vink 2006; Trundle et al. 2008; Gal-Yam \& Leonard 2009; Smith et al. 2007, 2008; Groh et al. 2013; Groh 2014; Boian \& Groh 2018). Most of the fundamental questions about the physical cause of the LBV instability remain unsolved. Hypotheses for the mechanism involve radiation pressure instabilities, turbulent pressure instabilities, vibrations and dynamical instabilities, and binarity (see Humphreys \& Davidson 1994 for an overview). The high stellar luminosities near the Eddington limit probably enable instabilities (several processes in the literature are summarized in Vink \& de Koter 2002; Owocki 2015; Guzik \& Lovekin 2014). The 
impact of binarity has received much attention lately (Gallagher 1989; Kashi 2010; Kashi \& Soker 2010; Smith 2011; Boffin et al. 2016). Binary scenarios for the formation of LBVs have been proposed. LBVs may be mass gainers that received a kick when the primary exploded or the product of a merger (e.g. Justham et al. 2014; Smith \& Tombleson 2015; Portegies Zwart \& van den Heuvel 2016). However, the mass-gainer scenario presented by Smith \& Tombleson (2015), on the basis of the apparent isolation of LBVs relative to massive star clusters, has been highly debated in the literature (e.g. Humphreys et al. 2016).

LBVs are surrounded by massive circumstellar nebulae of dust and gas, rich in processed material, indicative of stellar mass ejected by an evolved object through extensive stellar winds and outbursts. Representative examples in our Galaxy are the enigmatic $\eta$ Car (e.g. Morse et al. 1998; Smith et al. 2003; Smith 2013; Morris et al. 2017), AG Car (e.g. Thackeray 1950; Vamvatira-Nakou et al. 2015), HR Car (Hutsemekers \& van Drom 1991; Umana et al. 2009; Buemi et al. 2017), WRAY 15-751 (Hutsemekers \& van Drom 1991; Vamvatira-Nakou et al. 2013), AFGL 2298 (Ueta et al. 2001; Umana et al. 2005; Buemi et al. 2010), and the candidate LBVs HD 168625 (Umana et al. 2010, and reference therein), G79.29+0.46 (Rizzo et al. 2008, 2014; Umana et al. 2011a; Agliozzo et al. 2014), G26.47+0.02 (Clark et al. 2003; Umana et al. 2012; Paron et al. 2012), and the Pistol Star (Figer et al. 1999; Lang et al. 1999; Lau et al. 2014). The common presence of a N-enriched or dusty circumstellar nebula around LBVs allows the identification of candidate LBVs (e.g. Humphreys \& Davidson 1994; Nota et al. 1995; van Genderen 2001; Weis 2003), even if there is a lack of evidence of S Doradus variability. The discovery of an infrared ring nebula around Wray 17-96, identified by Egan et al. (2002) as a candidate LBV based on the mid-IR properties of the ejecta, is particularly important. Subsequently, the number of candidate LBVs increased considerably with mid-IR surveys carried out in the following years (Clark et al. 2005; Gvaramadze et al. 2010; Wachter et al. 2011; Flagey et al. 2014; Nowak et al. 2014).

Kochanek (2011) show that the physical requirement of dust condensation and growth in LBV ejecta (i.e. particle growth rate larger than grain photoevaporation rate) is met only for very high mass-loss rates $\left(>10^{-2.5} M_{\odot} \mathrm{yr}^{-1}\right)$. Such high massloss rates occur during LBV giant eruptions, when the stars form a cool $(\sim 7000 \mathrm{~K})$ and optically thick pseudo-photosphere that shields the dust from the soft UV photons and favours collisional particle growth with its high densities. Dust can also form close to periastron passage in colliding wind WC binaries, like in the case of HD 193793 (see Crowther 2007 for a review on dust formation in WR stars). A signature of wind-wind interaction with a companion star is a dusty "pinwheel" nebula (Tuthill et al. 2008). Interestingly, a dusty spiral nebula has been observed in the binary LBV HR Car (Boffin et al. 2016; Buemi et al. 2017).

In the last few years, Spitzer and Herschel observations have been critical to map the dust distribution in Galactic LBV nebulae, often suggesting episodic mass-loss events or revealing the presence of a photo-dissociation region (e.g. Umana et al. 2009, 2011b). Mapping the dust in LBV nebulae at lower metallicities, such as in the Magellanic Clouds (MCs), has not been possible so far, because of the limited angular resolution of available infrared (IR) instrumentation. With the Atacama Large Millimeter Array (ALMA), we can for the first time map the dust of LBV nebulae in nearby galaxies in the sub-millimetre (sub-mm) bands.

\section{The LBV status of RMC 143 and its circumstellar nebula}

\subsection{Is RMC 143 an LBV?}

RMC $143^{1}$ is classified as one of only eight confirmed LBVs in the Large Magellanic Cloud (LMC; Richardson \& Mehner 2018). The star lies in the outskirts of the 30 Doradus (hereafter 30 Dor) star-forming region (Walborn \& Blades 1997). Parker et al. (1993) classified the star as an LBV. The authors note photometric variations of at least $1.4 \mathrm{mag}$ in $V$ band. Spectroscopic observations indicate that the star changed from spectral type F5 to F8 in the 1950s, became as hot as an O9.5 star in 1981/85, before moving back to cooler temperatures and appearing as a late-B supergiant in 1992.

The original LBV classification of RMC 143 by Parker et al. (1993) is questionable as the large change in $V$-band photometry reported for 1981/85 and associated change in spectral type to late-O and also the F-type classifications in the 1950s and 1983 may be due to a misidentification of the star (Fig. 1):

1. There has been confusion in the literature between RMC 143 and HD 269929². In particular, Parker et al. (1993) state that HD 269929 is an alternative designation for RMC 143. HD 269929 has a $V$-band magnitude of 12.2 mag and shows no significant brightness variation between 2000 and 2009 in the All Sky Automated Survey (ASAS; Pojmanski 1997). The star is classified as F8 (SIMBAD; Cannon 1936), which matches the classification of RMC 143 in the 1950s and 1983.

2. Melnick (1985) classify stars in the centre of 30 Dor based on spectra obtained in 1983. They assign a spectral type F7Ia, in contradiction with the O-type star classification in 1981/85 of Parker et al. (1993). Their Fig. 1 identifies the correct star as RMC 143.

3. Clayton \& Martin (1985) exclude RMC 143 from their stellar sample to study interstellar dust in the LMC, as they were aware that different observers had observed two or three different stars.

4. The 1992 June International Ultraviolet Explorer (IUE) spectrum is consistent with a B8-9I star (Parker et al. 1993). From the FES image of the $I U E$ a $V$-band magnitude of 10.9 mag is estimated.

5. Walborn et al. (2017) report spectroscopic changes between the spectral types A and late-B in the time span from 1998 to 2016.

6. Photometry variations on time scales of several weeks and of the order of $0.5 \mathrm{mag}$ are observed in the ASAS lightcurve in the early 2000s. The All-Sky Automated Survey for SuperNovae (ASAS-SN) lightcurve shows no significant variations between 2014 and 2018 (Kochanek et al. 2017; Jayasinghe et al. 2018).

Unfortunately, the pre-1990 observations are not available to us. The F-type stellar classifications prior to 1990 may be observations of HD 269929 and the O-star classification in the early 1980s may also belong to observations of another star. However, the documented spectroscopic variations in Walborn et al. (2017) and the massive nebula (this work) both support an LBV classification. Given the star's relatively low luminosity for an LBV, small spectroscopic variabilities would be expected following the amplitude-luminosity relation of S Doradus variables (Wolf 1989).

\footnotetext{
1 RA 05:38:51.617, Dec -69:08:07.315 (ICRS coord., epoch=J2000). Alternate identifier: CPD-69 463.

2 RA 05:39:37.838, Dec -70:39:47.042 (ICRS coord., epoch=J2000).
} 


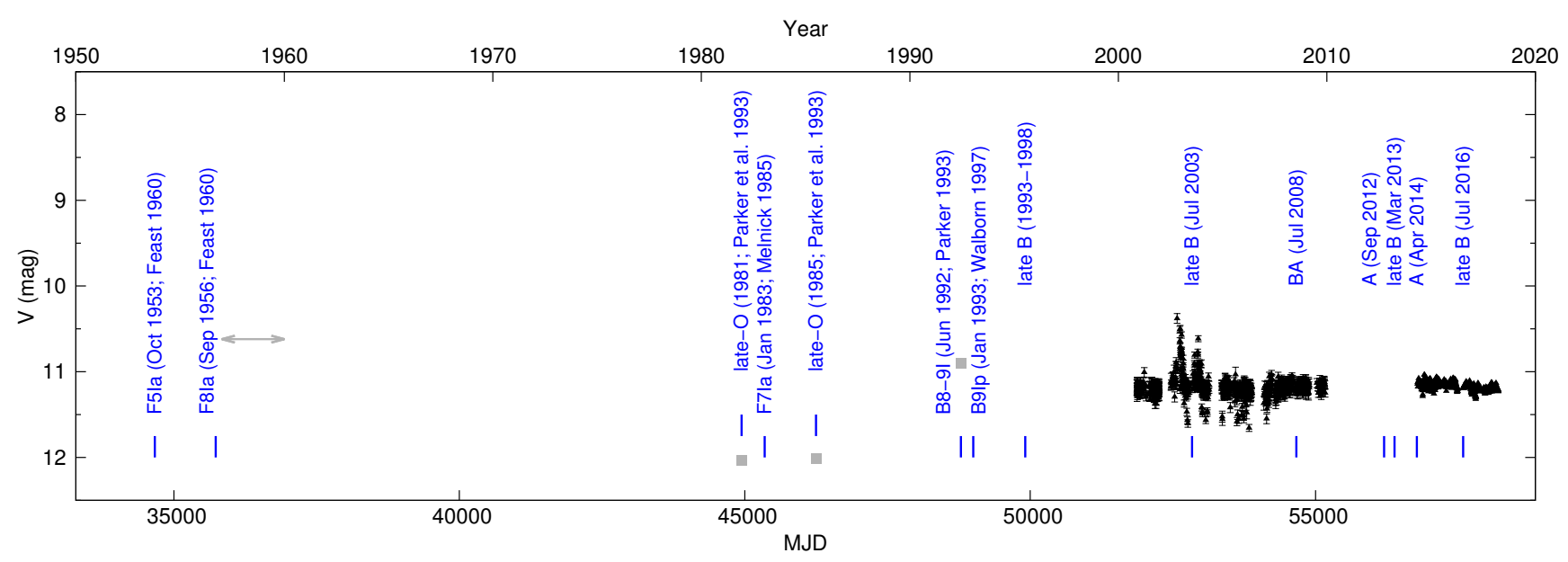

Fig. 1. Lightcurve of RMC 143. Historic photometry is displayed in grey, recent photometry by ASAS from 2000-2009 and ASAS-SN from 2014-2018 are displayed in black. The ASAS MAG_4 (6 pixel aperture) is used since smaller aperture contain many outliers due to background subtraction issues. We add $0.8 \mathrm{mag}$ to correct the magnitude, following Walborn et al. (2017). We indicate the reported spectral types in blue. When no reference is given, the value has been obtained from Walborn et al. (2017).

\subsection{The circumstellar nebula of RMC 143}

The identification of a circumstellar nebula around RMC 143 is difficult due to its location within the 30 Dor HII region. Feast (1961) noticed several nebular filaments, extending to an angular scale of $15^{\prime \prime}$. Smith et al. (1998) analysed the velocity field and abundances of these filaments and attributed them to the 30 Dor complex. Only a compact region up to about $2^{\prime \prime}$ from the star has abundances consistent with ejected stellar material during an LBV phase.

High spatial resolution Hubble Space Telescope (HST) images with the F656N filter show that the circumstellar material of RMC 143 is triangular shaped and is located north-west of the star (Weis 2003). The nebula is elongated with an extent of $4.9^{\prime \prime}$ (about $1.2 \mathrm{pc}$ ) and oriented north to south-west. Its shape is very unusual and raises the speculation that strong stellar winds or turbulent motion in the HII region caused its disruption. Highdispersion optical spectra confirm the presence of a nebula with two velocity components.

Agliozzo et al. (2012) presented the first radio observations of RMC 143, performed with the Australia Telescope Compact Array (ATCA) in Band $4 \mathrm{~cm}$. The resolution with the $6 \mathrm{~km}$ array configuration varied between $1.5^{\prime \prime}$ and $2.5^{\prime \prime}$, depending on the central observing frequency ( $9 \mathrm{GHz}$ and $5.5 \mathrm{GHz}$, respectively). This allowed to detect the radio counterpart of the optical nebula, which is ionized and emits in the radio by free-free transitions (bremsstrahlung emission). At $9 \mathrm{GHz}$, an unresolved source at the position of the star was detected. Comparing the radio and $\mathrm{H} \alpha$ flux densities, which trace the same gas, led to the conclusion that the optical nebula suffers intrinsic extinction due to dust.

In this paper, we present sub-mm/radio observations of RMC 143 with ALMA and ATCA and optical spectra with $\mathrm{X}$-shooter at the Very Large Telescope (VLT). We perform a multiwavelength study and compare our data to archival optical HST Wide Field and Planetary Camera 2 (WFPC2) images and IR photometry. We interpret the nature of the ALMA submillimetre emission.

\section{Observations}

\subsection{ALMA}

RMC 143 was observed with ALMA on 26 December 2014 as part of a Cycle-2 project (2013.1.00450.S, PI Agliozzo).
During the observations, forty $12 \mathrm{~m}$ antennas were used with an integration time of $16 \mathrm{~min}$. A standard Band 7 continuum spectral setup was used, resulting in four $2 \mathrm{GHz}$ width spectral windows of 128 channels of XX and YY polarization correlations centred at approximately 336.5 (LSB), 338.5 (LSB), 348.5 (USB), and $350.5 \mathrm{GHz}$ (USB). Atmospheric conditions were marginal for the combination of frequency and high airmass (transit elevation is $45^{\circ}$ for RMC 143 at the ALMA site). Non-standard calibration steps were required to minimize image degradation due to phase smearing, to provide correct flux calibration, and to maximize sensitivity by allowing inclusion of shadowed antennas. Further discussion of these techniques and general aspects of observations and data reduction can be found in Agliozzo et al. (2017a). We derived the intensity image from naturally weighted visibilities to maximize sensitivity and image quality (minimize the impact of phase errors on the longer baselines). We imaged all spectral windows together $(343.5 \mathrm{GHz}$ average; approximately $7.5 \mathrm{GHz}$ usable bandwidth), yielding an rms noise ${ }^{3}$ of $72 \mu \mathrm{Jy}_{\text {beam }}{ }^{-1}$. In Fig. 2 we show the 2, 3, and $4 \sigma$ contour levels of the emission. The lowest contour does not have strong statistical significance, but appears to partially overlap with the $\mathrm{H} \alpha$ emission. Deeper observations with ALMA would improve the image quality.

\subsection{ATCA}

We performed ATCA observations of RMC 143 between 20 and 23 January 2012, using the array in the most extended configuration $(6 \mathrm{~km})$ and the Compact Array Broadband Backend (CABB) " $15 \mathrm{~mm}$ " receiver in continuum mode. RMC 143 was observed at different hour angles distributed over three days, alternating with the phase calibrator ICRF J052930.0-724528. The observations were performed in marginal weather conditions, which may have caused a loss of coherence at the long baselines (small angular scales in the sky). For each source we split the receiver bandwidth in two $2 \mathrm{GHz}$ sub-bands, one centred at $17 \mathrm{GHz}$ and one at $23 \mathrm{GHz}$. The two data sets were reduced separately. Details on the observing strategy and data reduction can be found in Agliozzo et al. (2017a,b). Information

3 The originally desired rms noise of $40 \mu \mathrm{Jy} \mathrm{beam}^{-1}$ was not achieved because only one third of the required observations were completed. 


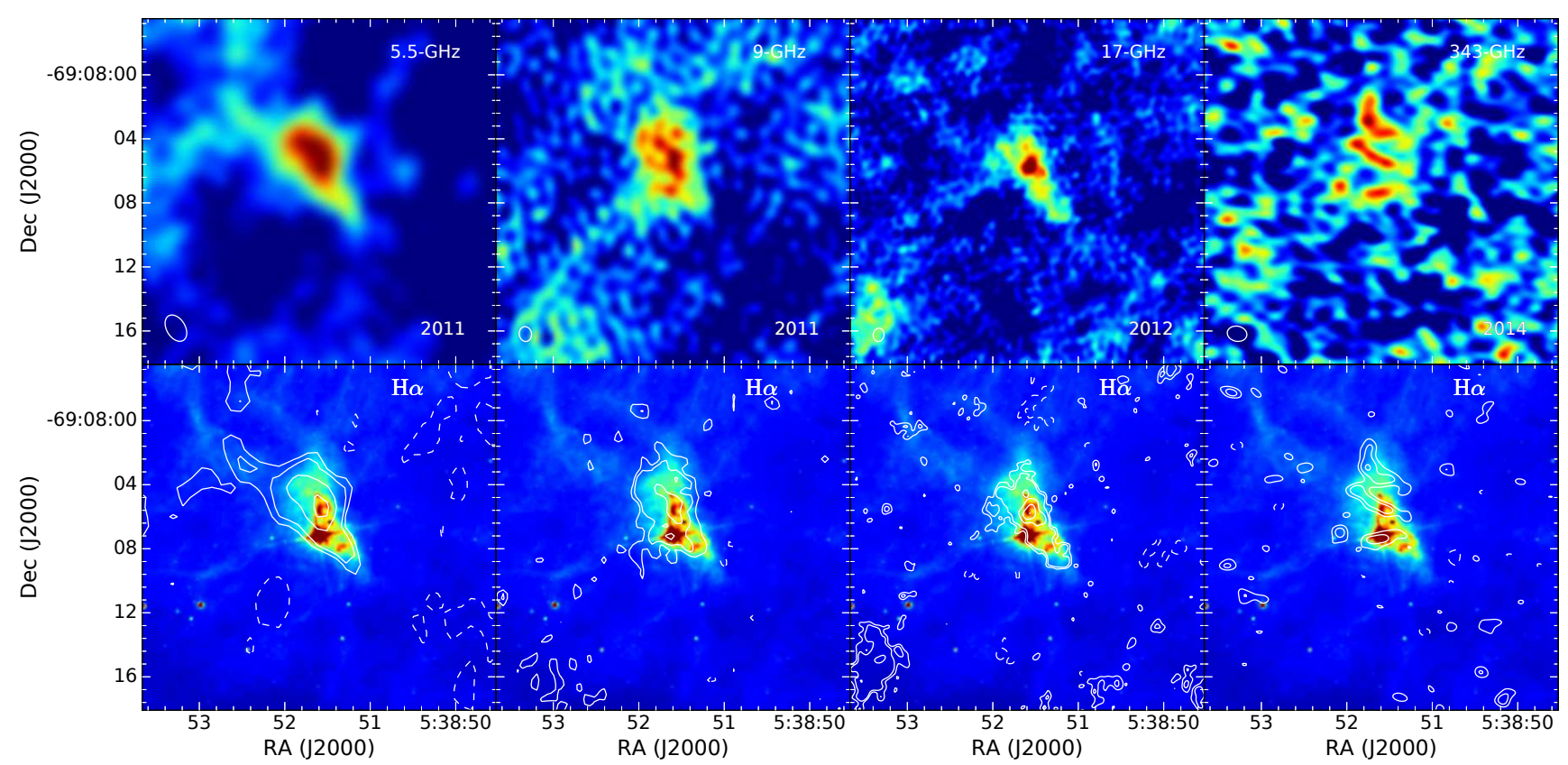

Fig. 2. From top-left to top-right: ATCA 5.5, 9 and $17 \mathrm{GHz}$, ALMA $343 \mathrm{GHz}$ maps, showing the same field of view. The synthesized beam is indicated as a white ellipse. From bottom-left to bottom-right: radio and sub-mm contours superimposed on the HST H $\alpha$ image (Weis 2003). The lowest contour level is $2 \sigma$, followed by $3 \sigma$ and then increases in steps of three (steps of two at $9 \mathrm{GHz}$ ). In the case of the $343 \mathrm{GHz}$ map, the 2,3 and $4 \sigma$ levels are shown. All the panels are centred on the stellar position, which is the brightest source in the $\mathrm{H} \alpha$ image.

Table 1. Date of observation, interferometer, central frequency, largest angular scale, synthesis beam, position angle, peak flux density $F_{v}$, and spatially-integrated flux density $S_{v}$.

\begin{tabular}{lccccccc}
\hline \hline Date & Array & $\begin{array}{c}\text { Frequency } \\
(\mathrm{GHz})\end{array}$ & $\begin{array}{c}\text { LAS } \\
\left({ }^{\prime \prime}\right)\end{array}$ & $\begin{array}{c}\text { HPBW } \\
\left({ }^{\prime \prime 2}\right)\end{array}$ & $\begin{array}{c}\text { PA } \\
\left({ }^{\circ}\right)\end{array}$ & $\begin{array}{c}F_{v} \\
\left(\mathrm{mJy} \mathrm{beam}^{-1}\right)\end{array}$ & $\begin{array}{c}S_{v} \\
\left(\mathrm{mJy} \text { beam }^{-1}\right)\end{array}$ \\
\hline $2011-04-18 / 20$ & ATCA & 5.5 & $6.5^{(a)}$ & $1.98 \times 1.60^{(a)}$ & -178 & $0.51 \pm 0.05$ & $1.3 \pm 0.3$ \\
$2011-04-18 / 20$ & ATCA & 9 & $6.5^{(a)}$ & $1.30 \times 1.04^{(a)}$ & -175 & $0.28 \pm 0.05$ & $1.5 \pm 0.5$ \\
$2012-01-21 / 23$ & ATCA & 17 & 6.5 & $0.84 \times 0.68$ & -8.8 & $0.192 \pm 0.016$ & $1.1 \pm 0.1$ \\
$2012-01-21 / 23$ & ATCA & 23 & 4.1 & $0.63 \times 0.51$ & -8.8 & $<0.15$ & - \\
$2014-12-26$ & ALMA & 343 & 9.0 & $1.23 \times 0.95$ & 78.7 & $0.300 \pm 0.072$ & $2.5 \pm 0.4$ \\
\hline
\end{tabular}

Notes. ${ }^{(a)}$ After filtering out $u v<17 \mathrm{k} \lambda$ visibilities.

on the final images, obtained by adopting the natural weighting scheme of the visibilities, can be found in Table 1 . The table also lists information about the synthesized beam (Half Power Beam Width, HPBW), position angle (PA), largest angular scale (LAS), peak flux density, and rms-noise.

At $17 \mathrm{GHz}$, we detect above $3 \sigma$ the nebular emission in its whole optical extent. At $23 \mathrm{GHz}$, the map is noisy because of the system response to bad weather at higher frequencies and we did not detect with statistical significance emission associated with RMC 143. We thus do not show the $23 \mathrm{GHz}$ data.

We include in our analysis the 5.5 and $9 \mathrm{GHz}$ data from the ATCA observations performed in 2011 with the CABB " $4 \mathrm{~cm}$ Band" (4-10.8 GHz) receiver, described in Agliozzo et al. (2012) and shown in Fig. 2. At these frequencies the HII region is bright and causes confusion. To mitigate artefacts due to the secondary lobes from bright sources in the region, we reprocessed the data and filtered out the $u v$ spacings smaller than $\sim 17 \mathrm{k} \lambda$, in order to match the LAS of the $17 \mathrm{GHz}$ data. This operation degrades the image quality and the rms-noise. In Table 1, the LAS and beam information at 5.5 and $9 \mathrm{GHz}$ refer to the newly processed maps, which are used to derive the spatially-integrated flux density.

\section{3. $V L T / X$-shooter}

RMC 143 was observed with X-shooter at the VLT on 2 October 2015 and 12 January 2016 (ESO programme 096.D-0047(A), PI Mehner). X-shooter is a medium-resolution echelle spectrograph that simultaneously observes the wavelength region from 3000-24800 A with three arms (Vernet et al. 2011). Spectra were obtained with the narrowest available slits of 0.5 in the UVB arm, 0.' 4 in the VIS arm, and 0.' 4 in the NIR arm yielding spectral resolving powers of $R \sim 9000-17000$. Spectra obtained with the $5^{\prime \prime}$ slits provide us the means to achieve absolute flux calibrations to $\sim 20 \%$ accuracy. However, the large aperture includes nebular reflection. The data were reduced with the ESO X-shooter pipeline version 3.2.0, and the flux normalization was computed using custom IDL routines developed by one of us (JHG). We estimate that the uncertainty in the flux normalization is about $2 \%$.

\subsection{HST}

The HST images were obtained with the WFPC2 instrument using the $\mathrm{H} \alpha$-equivalent filter F656N (proposal ID 6540, PI 
Schulte-Ladbeck). The images were retrieved from the STScI data archive, combined, and astrometrically recalibrated as described in Agliozzo et al. (2012). These data were first published by Weis (2003).

\section{The stellar parameters and atmosphere abundances of RMC 143}

Many emission lines form in the dense stellar winds of LBVs and veil the underlying photospheric spectrum. Complex radiative transfer models, which include the necessary physics to study the radiation transport across the atmosphere and wind, are needed to obtain realistic parameters. We compare the X-shooter spectra of RMC 143 obtained in 2015 with atmosphere models computed with the radiative transfer code CMFGEN (version 5 May 17, Hillier \& Miller 1998), which has been successfully applied to several LBVs (e.g. Hillier et al. 2001; Groh et al. 2006, 2009, 2011; Clark et al. 2009, 2012; Georgiev et al. 2011; Mehner et al. 2017). For a review on the essential properties of the code and the basics of spectroscopic analysis of massive stars using photospheric and wind diagnostics, see Groh (2011) and Martins (2011).

The diagnostics to find the best-fitting stellar model for RMC 143 are similar to those described in Mehner et al (2017), except for the effective temperature. This quantity is constrained based on the equivalent widths of the $\mathrm{Mg}$ II $\lambda 4481$ and $\mathrm{Mg}$ I $\lambda 5183$ lines. The value of $T_{\text {eff }}$ is supported by $\mathrm{He}_{\mathrm{I}} \lambda 4713$, He 1 15876, photospheric Fe II lines, and the emission components of $\mathrm{Fe}$ II lines formed in the wind. The mass-loss rate is constrained based on the emission components of Fe II and $\mathrm{H}$ Balmer lines. We note that in this parameter regime, the strength of the $\mathrm{H}$ emission lines is extremely sensitive on the effective temperature and temperature structure of the wind. The luminosity of RMC 143 is constrained based on the absolute flux calibration of the X-shooter spectrum used to constrain the other stellar parameters. Since RMC 143 is a variable star, we consider this more reliable than using broad-band photometry obtained at different epochs.

Figure 3 compares the 2015 X-shooter spectrum of RMC 143 with our best-fit CMFGEN model. The derived stellar parameters for its quiescent state are listed in Table 2. We find an effective temperature of $T_{\text {eff }}=8500 \pm 300 \mathrm{~K}$ and a stellar luminosity of $\log \left(L / L_{\odot}\right)=5.32 \pm 0.05\left(M_{\mathrm{bol}}=-8.56 \mathrm{mag}\right)$. We note that the value of $T_{\text {eff }}$ derived from the $\mathrm{Mg}_{\mathrm{I}} / \mathrm{Mg}_{\text {II }}$ line ratio does not provide enough free electrons in the wind, which are needed for producing the electron scattering wings clearly visible in the $\mathrm{H}$ recombination lines. In addition, the $\mathrm{H}$ ionization structure seems to require a slightly higher value of $T_{\text {eff }}$. In this parameter range, our models show that a small increase of $T_{\text {eff }}$ (by $500-800 \mathrm{~K}$ ) would be enough for producing stronger $\mathrm{H}$ emission and broader electron scattering wings. We still prefer to give higher weight to the $\mathrm{Mg} / \mathrm{I} / \mathrm{Mg}$ II, since these lines are formed deeper in the wind. Our interpretation is that the $\mathrm{H}$ ionization structure is not fully reproduced by our models, and is likely affected by time-dependent effects that are inherent to LBVs (Groh \& Vink 2011).

Our derived value of $\log \left(L / L_{\odot}\right)=5.32$ is lower than the most commonly-used estimate $\left(\log \left(L / L_{\odot}\right)=5.7\right)$, which was suggested by Parker et al. (1993). However, our luminosity value is in line with the estimate from Walborn \& Blades (1997), who originally suggested that R143 had an initial single-star mass around $25-30 M_{\odot}$. The two most likely possibilities are that (1) Parker et al. (1993) misidentified RMC 143 (see Sect. 2), or (2) RMC 143 had a different value of luminosity and temperature
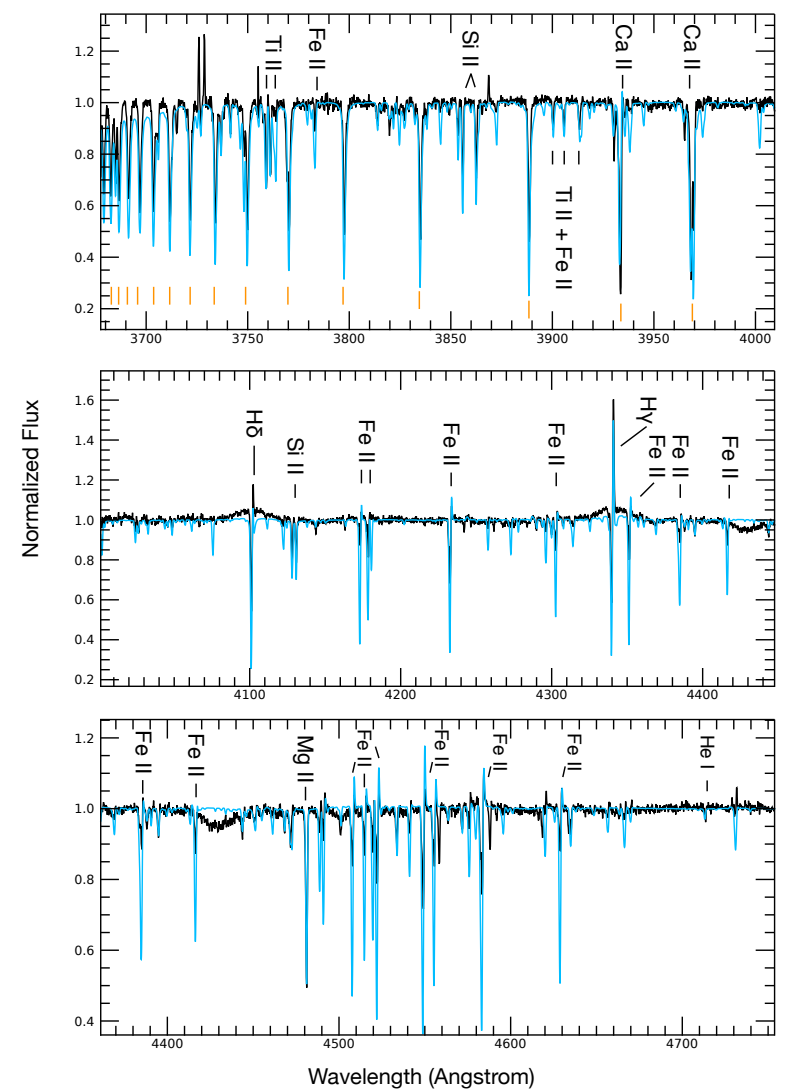

Fig. 3. 2015 X-shooter spectrum of RMC 143 (black line) and bestfit CMFGEN model (blue line). The spectral ranges cover important hydrogen and helium lines, but also $\mathrm{Si}$ II, $\mathrm{Mg}$ II, Fe II, and $\mathrm{O}_{\text {I. }}$ The orange ticks correspond to hydrogen lines.

around the early 1990s. Figure 4 shows the position of RMC 143 in the HR diagram obtained from our CMFGEN modelling.

Extended emission from surrounding material is apparent in the spectra and not all hydrogen and helium lines are matched equally well. The estimation of the effective temperature $T_{\text {eff }}$ is not affected by the nebular contamination, while the luminosity $L$ may be somewhat overestimated due to inclusion of reflected or scattered nebular continuum. Since the hydrogen absorption lines are filled in with extra emission from the surrounding nebula, we only derive a lower limit of the terminal wind velocity $v_{\infty}>70 \mathrm{~km} \mathrm{~s}^{-1}$.

The gravity is estimated to be $\log (g /[\mathrm{cgs}])=0.7$ and the stellar mass to be $\sim 8 M_{\odot}$. A mass-loss rate of $\dot{M} \sim 1.0 \times 10^{-5} M_{\odot} \mathrm{yr}^{-1}$ for a clumping factor $f_{\mathrm{c}}$ equal to 1 fits the hydrogen emission well. The mass-loss rate is more affected by the uncertain ionization structure and time dependence than by nebular contamination since we used mainly $\mathrm{H}_{\beta}$, higher Balmer lines, and $\mathrm{Fe}$ II lines to constrain it. For comparison, the LBV W243 with a similar temperature of $T_{\text {eff }} \sim 8500 \mathrm{~K}$ but much higher luminosity has a much weaker mass-loss rate of $6.1 \times 10^{-7} M_{\odot} \mathrm{yr}^{-1}$ (Ritchie et al. 2009).

RMC 143 displays the spectrum of an LBV. In particular, the spectrum displays no [O I] $\lambda \lambda 6300,6364$ emission, characteristic of the $\operatorname{sgB}[\mathrm{e}]$ class (Humphreys et al. 2017). We also detect no $\mathrm{CO}$ first-overtone emission at $2.3 \mu \mathrm{m}$, indicative that RMC 143 has no high density circumstellar disc as is the case for $\mathrm{B}$ [e] stars (McGregor et al. 1988a). RMC 143 shows signs of CNOprocessed material at the surface (Table 3), with enhanced $\mathrm{He}$ and $\mathrm{N}$, and depleted $\mathrm{H}, \mathrm{C}$, and $\mathrm{O}$. This confirms the evolved nature of the star, especially given the low current mass $\left(\sim 8 M_{\odot}\right)$. 

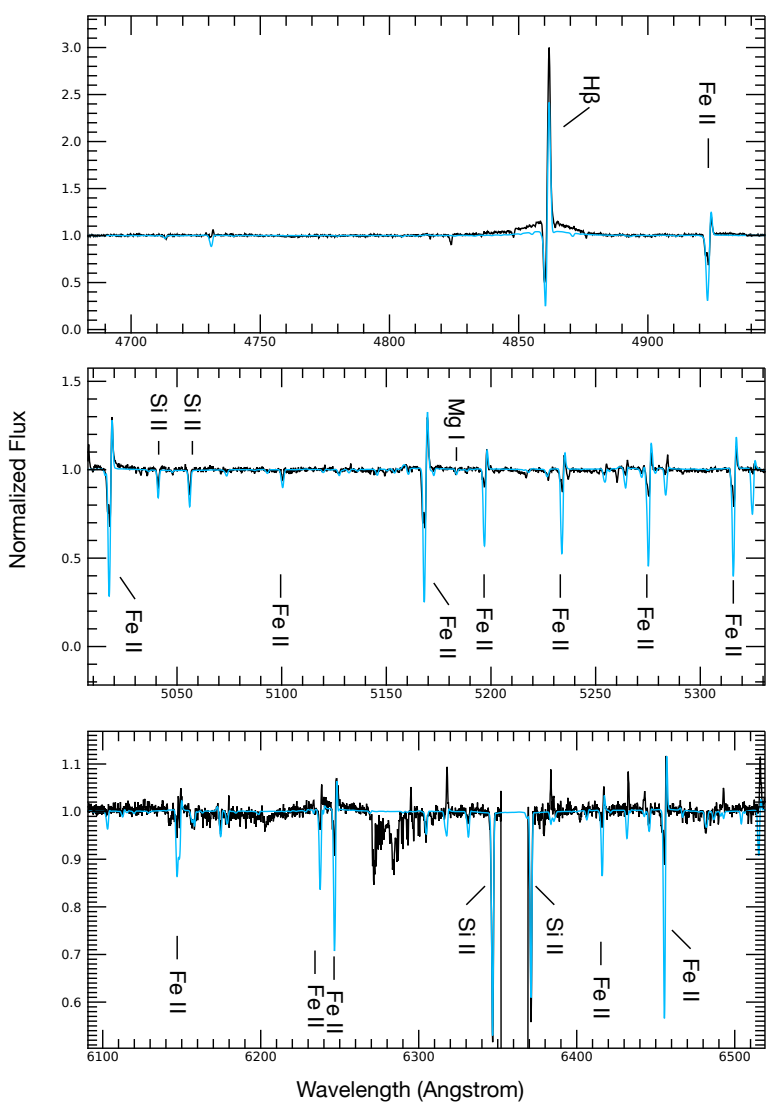

Fig. 3. continued.

Table 2. Fundamental stellar parameters of RMC 143 from the best-fit CMFGEN model of X-shooter spectra on 2 October 2015.

\begin{tabular}{lcc}
\hline \hline Parameter & Value & Error \\
\hline$T_{\text {eff }}(a)$ & $8500 \mathrm{~K}$ & $300 \mathrm{~K}$ \\
$T_{*}(b)$ & $9600 \mathrm{~K}$ & $1000 \mathrm{~K}$ \\
$\log \left(L_{*} / L_{\odot}\right)$ & 5.32 & 0.05 \\
$M_{\text {bol }}$ & -8.56 & 0.04 \\
$\dot{B C}_{V}$ & -0.14 & 0.02 \\
$\dot{M}^{(c)}$ & $1.0 \times 10^{-5} M_{\odot} \mathrm{yr}^{-1}$ & $0.2 \times 10^{-5} M_{\odot} \mathrm{yr}^{-1}$ \\
$\log (g /[\mathrm{cgs}])^{(d)}$ & 0.7 & 0.1 \\
$M$ & $\sim 8.2 M_{\odot}$ & \\
$R_{\text {phot }}$ & $\sim 211 R_{\odot}$ & \\
$R_{*}$ & $\sim 169 R_{\odot}$ & \\
$v_{\infty}$ & $70 \mathrm{~km} \mathrm{~s}^{-1}$ & $10 \mathrm{~km} \mathrm{~s}^{-1}$ \\
$E(B-V)$ & $0.42 \mathrm{mag}$ & $0.02 \mathrm{mag}$ \\
$R_{V}$ & 4.0 & 0.1 \\
\hline
\end{tabular}

Notes. ${ }^{(a)}$ Parameters labelled "eff" and "phot" refer to $\tau_{\text {ROSS }}=2 / 3$. ${ }^{(b)}$ Parameters labelled "*" refer to $\tau_{\text {ROsS }}=20$. ${ }^{(c)}$ The clumping factor $f_{\mathrm{c}}$, which is unknown, is set to unity. ${ }^{(d)}$ Specified at $\tau_{\text {ROsS }}=2 / 3$.

\section{Morphology of the radio and sub-millimetre emission}

RMC 143 lies south-east from the centre of the 30 Dor starforming region, the most luminous HII region in the Local Group. The environment around RMC 143 is rich in interstellar material and hot luminous stars and is very bright in the optical, IR, and radio. Figure 5 shows part of the 30 Dor complex
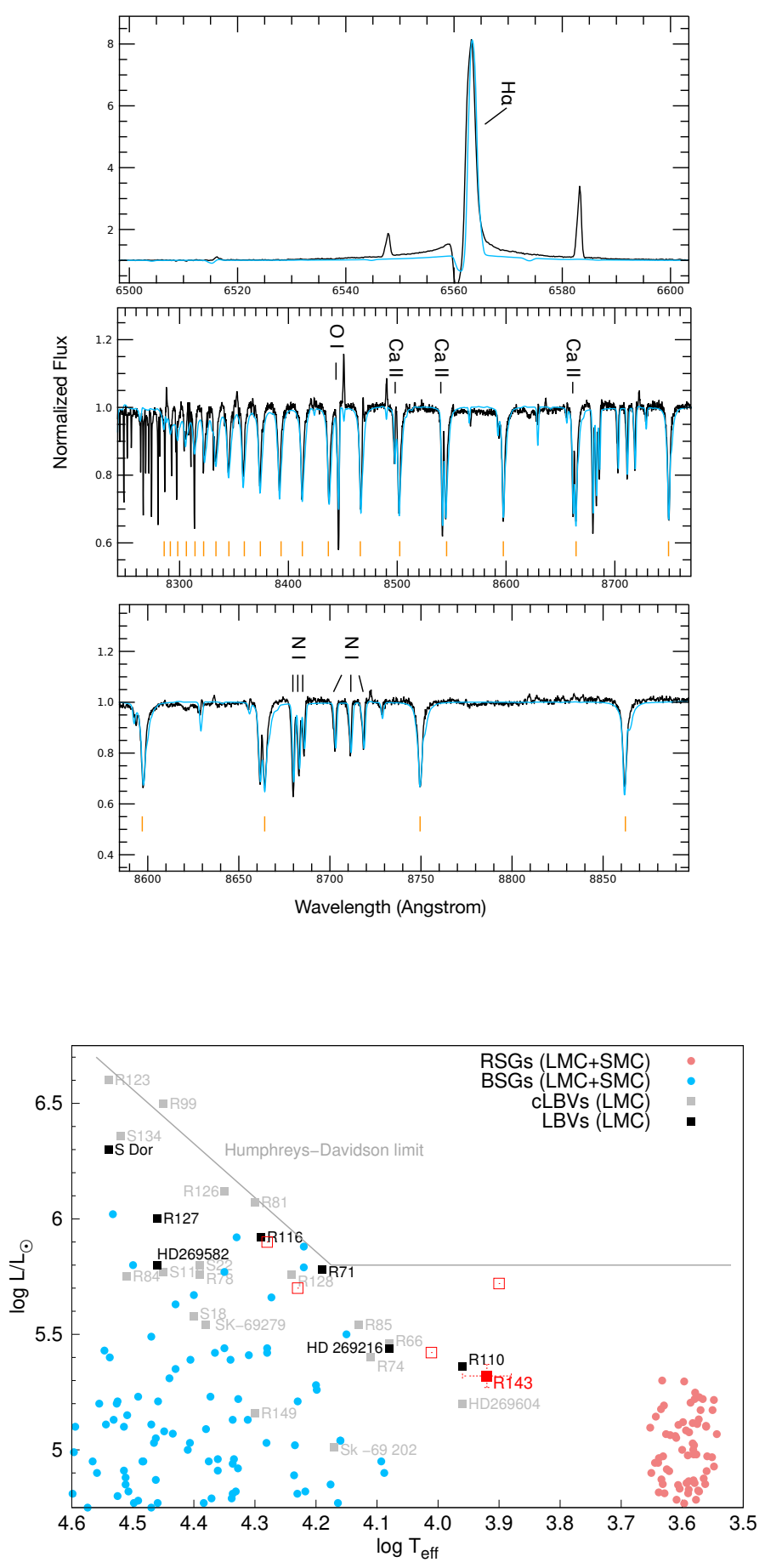

Fig. 4. Schematic upper HR diagram of the LMC, adapted from Mehner et al. (2017). Confirmed LBVs (black) and candidate LBVs (grey) are shown at their minimum phases. Blue and red supergiants are indicated with blue and red filled circles. We show the location of RMC 143 obtained in this work (filled red square). Previous literature values for RMC 143 are also shown (open red squares).

as seen by ATCA in the $6-\mathrm{km}$ configuration at $5 \mathrm{GHz}$. The LAS in this map is $\sim 20^{\prime \prime}$, which means that more extended radio emission in the star-forming region is filtered out by the interferometer. The Feast filaments (with an angular scale of $\sim 15^{\prime \prime}$ ) are not detected at $5 \mathrm{GHz}$, probably because they are too faint, although the $2 \sigma$ contour elongated toward the N-E side of the nebula suggests a partial detection of one of the 
Table 3. Surface chemical abundances for RMC 143 derived from the $2015 \mathrm{X}$-shooter spectrum.

\begin{tabular}{cccc}
\hline \hline Species & $\begin{array}{c}\text { Number fraction } \\
\text { (relative to H) }\end{array}$ & Mass fraction ${ }^{(a)}$ & $Z / Z_{\mathrm{LMC}}$ \\
\hline $\mathrm{H}$ & 1.0 & $4.3 \mathrm{e}-01$ & 0.58 \\
$\mathrm{He}$ & $3.3 \mathrm{e}-01$ & $5.6 \mathrm{e}-01$ & 2.19 \\
$\mathrm{C}$ & $4.0 \mathrm{e}-05$ & $2.0 \mathrm{e}-04$ & 0.20 \\
$\mathrm{~N}$ & $7.5 \mathrm{e}-04$ & $4.2 \mathrm{e}-03$ & 14.89 \\
$\mathrm{O}$ & $1.5 \mathrm{e}-04$ & $1.0 \mathrm{e}-03$ & 0.41 \\
\hline
\end{tabular}

Notes. Error estimates are on a $20 \%$ level. ${ }^{(a)}$ LMC mass fraction used: $\mathrm{H}=7.38 \mathrm{e}-01, \mathrm{He}=2.56 \mathrm{e}-01, \mathrm{C}=9.79 \mathrm{e}-04, \mathrm{~N}=2.82 \mathrm{e}-04, \mathrm{O}=2.45 \mathrm{e}-$ 03 .

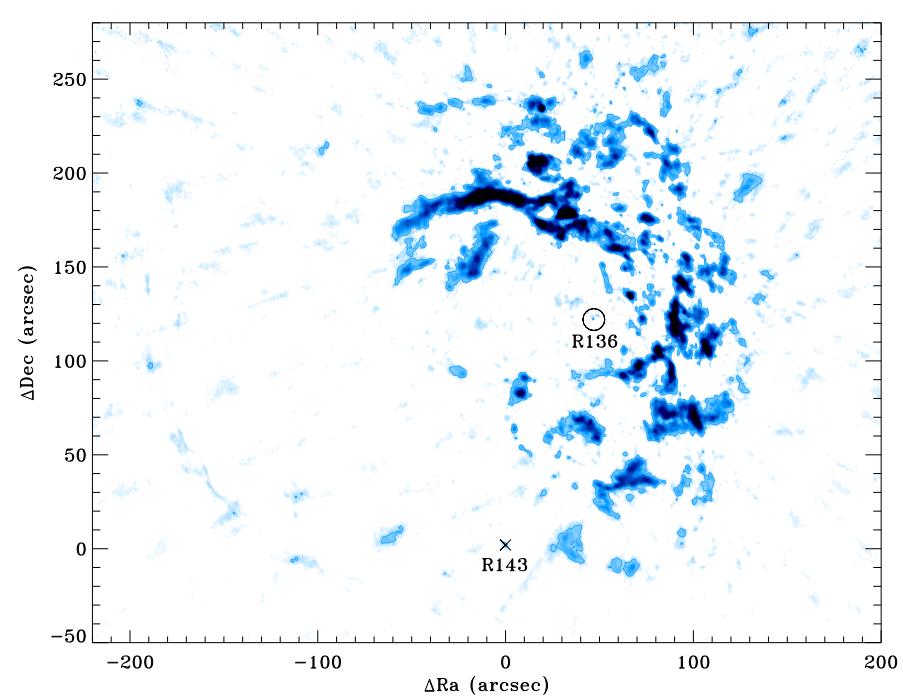

Fig. 5. ATCA $5 \mathrm{GHz}$ map from the observations presented in Agliozzo et al. (2012), showing the 3- $\sigma$ contours. The cross indicates the position of RMC 143, which is the phase centre of the field, and the circle the position of the stellar cluster RMC 136. The primary beam FWHM is about 9 arcmin.

filaments. At higher frequencies, the filaments would be filtered out because of the lack of baselines for those angular scales. The Feast filaments and RMC 143's nebula are illustrated in the $\mathrm{H} \alpha$ image of Fig. 6, with the ALMA contours at $343 \mathrm{GHz}$ overlaid.

As is the case in the optical, the nebula is also triangularshaped in the radio, with no counterpart in the east and southeast of the star (Fig. 2). From the $17 \mathrm{GHz}$ map, which has the highest spatial resolution, we estimate a size (beam convolved) of the radio emission of about $6^{\prime \prime}$ along the largest extension. The point-source detected at $9 \mathrm{GHz}$ at the position of the star is not detected at $17 \mathrm{GHz}$, most likely because of lack of coherence at the smallest angular scales due to high phase-rms during the observations. The knot of bright emission visible at all radio frequencies corresponds to the nebular material $2^{\prime \prime}$ north of the star, attributed to RMC 143 (Smith et al. 1998).

The sub-mm emission detected by ALMA has an irregular shape and is distributed on one side of the star (Fig. 6), similarly to the optical and radio emissions. However, the sub-mm contours suggest a different morphology and thus a different nature of the emission. The sub-mm continuum emission can be due to both free-free emission from the ionized gas and to thermal emission from dust. The slightly curved emission in the sub-mm seems aligned with an "imaginary" extension of at least one of

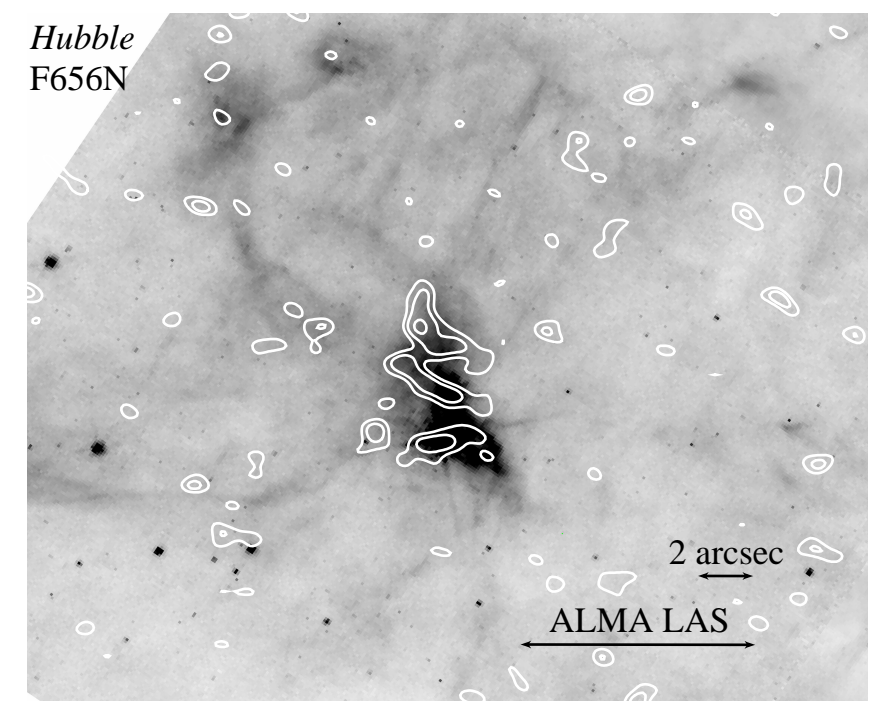

Fig. 6. HST/H $\alpha$ image (Weis 2003), overlaid with the 2, 3, $4 \sigma$ contours of the ALMA map at $343 \mathrm{GHz}$. North is up and east is left.

the Feast filaments over RMC 143's nebula. This is not seen in the radio maps. A compact object at the position of the star is also detected in the ALMA map with a statistical significance of $4 \sigma$.

Ideally, we would like to compare the sub-mm continuum emission with far-IR images. Unfortunately, the spatial resolution of IR space telescopes is unsuitable for disentangling the contribution of the central star, the circumstellar nebula, and the interstellar dust from the total emission. For example, the Spitzer/MIPS $24 \mu \mathrm{m}$ image has a resolution of $\sim 6^{\prime \prime}$, too poor for morphological comparison with other wavelengths. We retrieved the $8 \mu \mathrm{m}$ IRAC image (Meixner et al. 2006, with a resolution of $\sim 2^{\prime \prime}$ ) and subtracted the point source at the position of RMC 143 using the tool APEX in the MOPEX package (Makovoz \& Marleau 2005; NASA/IPAC Infrared Science Archive et al. 2011). The point-source subtraction worked relatively well considering the high and not uniform background, and the residual image is sufficient for a qualitative comparison with the ALMA and ATCA data. The spatial resolution is still poor and the central part of the nebula may have been subtracted together with the point-source. However, it is possible to recognise an outer asymmetric emission that seems to overlap better with the submm $343 \mathrm{GHz}$ ALMA contours than the $\mathrm{cm} 17 \mathrm{GHz}$ ATCA contours (Fig. 7). Thus, the $8 \mu \mathrm{m}$ flux plausibly arises from the same component responsible for the ALMA contours.

\section{Ionized matter}

The flux density of RMC 143 and the rms-noise $\sigma$ is determined from the $17 \mathrm{GHz}$ radio map using the CASA viewer. For the flux density, we integrate the nebular emission of areas above $3 \sigma$ level. The rms-noise is evaluated in regions free of emission and the flux density error is estimated as $\epsilon=\sigma \sqrt{N}$, where $N$ is the number of independent beams in the selected region. Calibration errors are negligible and the uncertainty is dominated by the noise in the maps and possible systematic under-estimated due to the $3 \sigma$ cut. We also estimate the flux densities at 5.5 and $9 \mathrm{GHz}$ from the reprocessed maps (Sect. 3.2) to reduce the contribution from the HII region extended emission and/or image artefacts due to zero-baseline scale missing (Table 1; compare with Agliozzo et al. 2012). In fact, both can contaminate the 


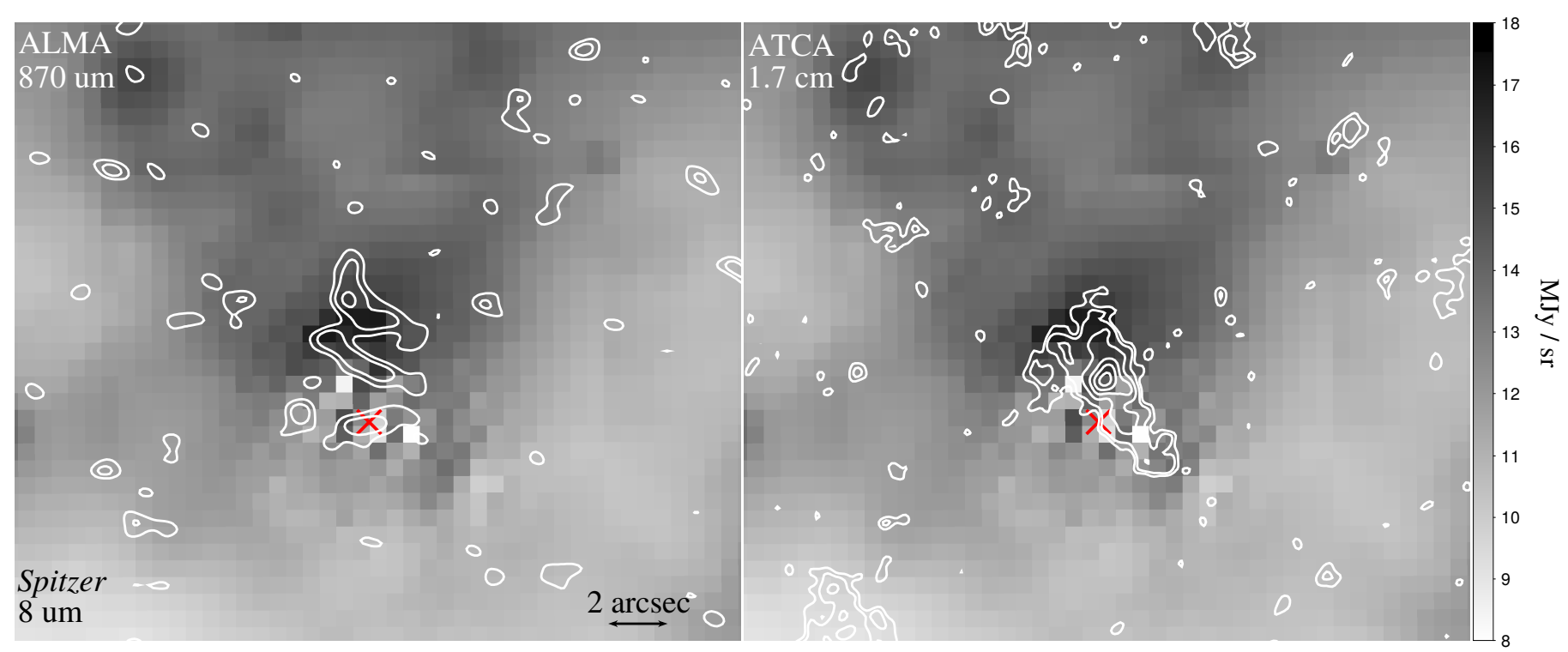

Fig. 7. Residuals from the $8 \mu \mathrm{m}$ IRAC image after point-source subtraction. The white contours are the [2, 3, $4 \sigma$ ] ALMA Band 7 emission (left) and the $[2,3,4,5,6,7 \sigma]$ ATCA $17 \mathrm{GHz}$ emission (right). North is up and east is left. The red cross indicates the position of the star.

nebular emission and eventually falsify the flux density analysis. Because of the inhomogeneous background in the 5.5 and $9 \mathrm{GHz}$ maps, the flux density measurements have a larger uncertainty, especially at $9 \mathrm{GHz}$.

The total integrated flux density encompassing the nebula and the central object at $343 \mathrm{GHz}$ is $2.5 \pm 0.4 \mathrm{mJy}$ (the error also includes $10 \%$ flux uncertainty). Part of this emission probably originates from the ionized nebula and is due to bremsstrahlung. In fact, from the flux density at $17 \mathrm{GHz}$, the extrapolated value at $343 \mathrm{GHz}$ accounting for the free-free emission is $\sim 0.8 \mathrm{mJy}$, about one third of the observed total flux density at $343 \mathrm{GHz}$.

We derived a spectral index map (per-pixel) between 5.5 and $17 \mathrm{GHz}$, after re-gridding the highest-resolution map $(17 \mathrm{GHz})$ to the same grid of the $5.5 \mathrm{GHz}$ map and re-convolved it with a synthesized beam that matches the resolution at $5.5 \mathrm{GHz}$, similar to Agliozzo et al. (2017b). Figure 8 shows the spectral index map and its associated error map. Calibration errors are negligible and the error in each pixel is given by the sum in quadrature of the rms-noise in both the maps. The weighted fit of the powerlaw $S_{v} \propto v^{\alpha}$ between the flux densities at 5.5, 9, and $17 \mathrm{GHz}$ gives us a mean spectral index $\langle\alpha\rangle=-0.2 \pm 0.2$, which is consistent with optically thin free-free emission typical of evolved HII regions (theoretically it is $S_{v} \propto v^{-0.1}$ ). The knot visible at all the radio frequencies in the nearly-middle part of the nebula has a flat flux density distribution (spectral index $\alpha \approx 0$ ), typical of optically thin bremsstrahlung emission. This suggests that its large brightness relative to the surrounding nebula is due to additional material along the line of sight rather than a density clump.

The point-source at the position of the star visible at 9 and $343 \mathrm{GHz}$ has peak flux densities of $0.20 \pm 0.05 \mathrm{mJy}^{-1}$ beam $^{-1}$ and $0.27 \pm 0.07 \mathrm{mJy} \mathrm{beam}^{-1}$, respectively. We use the stellar parameters derived in Sect. 4 and the formulation of the spectrum of free-free radiation from ionized spherical stellar winds by Panagia \& Felli (1975) to determine the expected flux density at 9, 17 and $343 \mathrm{GHz}$, as

$$
\begin{aligned}
S_{v}= & \times 10^{10} v_{[\mathrm{GHz}]}^{0.6} T_{\mathrm{e}[\mathrm{K}]}^{0.1}\left(\dot{M}_{\left[M_{\odot} \mathrm{yr}^{-1}\right]} \sqrt{f_{\mathrm{c}}}\right)^{4 / 3}\left(v_{\infty\left[\mathrm{km} \mathrm{s}^{-1}\right]} \times \mu\right)^{-4 / 3} \\
& \times \bar{Z}^{2 / 3} D_{[\mathrm{kpc}]}^{-2}[\mathrm{mJy}],
\end{aligned}
$$

where we assume a mean molecular weight per ion $\mu$ equal to 1.33 (adopting the He abundance determined in Sect. 4), an electron temperature of $T_{\mathrm{e}}=5000 \mathrm{~K}$ and an average ionic charge of $\bar{Z}=0.9$, accounting for the fact that in such a cool star He should be neutral. Same values for $T_{\mathrm{e}}$ and $\bar{Z}$ were used by Leitherer et al. (1995) to model the radio emission of Cygnus OB2 No. 12. We set $f_{\mathrm{c}}$ equal to 1 . With these assumptions we find flux densities of $0.45_{-0.10}^{+0.13} \mathrm{mJy}, 0.07 \pm 0.02 \mathrm{mJy}$ and $0.05_{-0.01}^{+0.02} \mathrm{mJy}$ at 343,17 and $9 \mathrm{GHz}$, respectively. The errors were derived taking into account the uncertainties from the CMFGEN model and a $10 \%$ uncertainty for $\bar{Z}$ and $\mu$. Based on this model, at $343 \mathrm{GHz}$ we would expect to observe a higher flux density; at $9 \mathrm{GHz}$ the measurement might include some nebular flux; at $17 \mathrm{GHz}$ we might have lost the detection of the point source because of decorrelation during the observations due to unstable weather. However, the terminal velocity $v_{\infty}$ determined in Sect. 4 is a lower limit, thus the above flux densities must be treated as upper limits.

It seems plausible that the sub-mm emission is due to free-free, but such a model of stationary, isothermal and spherically symmetric optically thick ionized wind may not applicable to RMC 143. The ionization fraction in the wind might be lower than assumed, given the low effective temperature and the hydrogen in the wind might recombine at large distances from the surface, among many possible scenarios. For RMC 127, Agliozzo et al. (2017a) found that a collimated ionized wind model better explains the observed SED from the near-IR to the radio. Other measurements in between the two ALMA and ATCA frequencies and between the near-IR and the sub-mm would be very useful to better understand the nature of the central object emission. Another source of emission in the sub-mm could be dust in the wind or in a shell in front of the star.

\section{The spatial distribution of dust}

The $\mathrm{H} \alpha$ emission in the nebula of RMC 143 is due to the de-excitation of the recombined hydrogen atom and the radio centimetric emission is due to free-free transitions. They trace the same gas (Agliozzo et al. 2012) and it is thus possible to determine the extinction of the $\mathrm{H} \alpha$ line by comparing the 


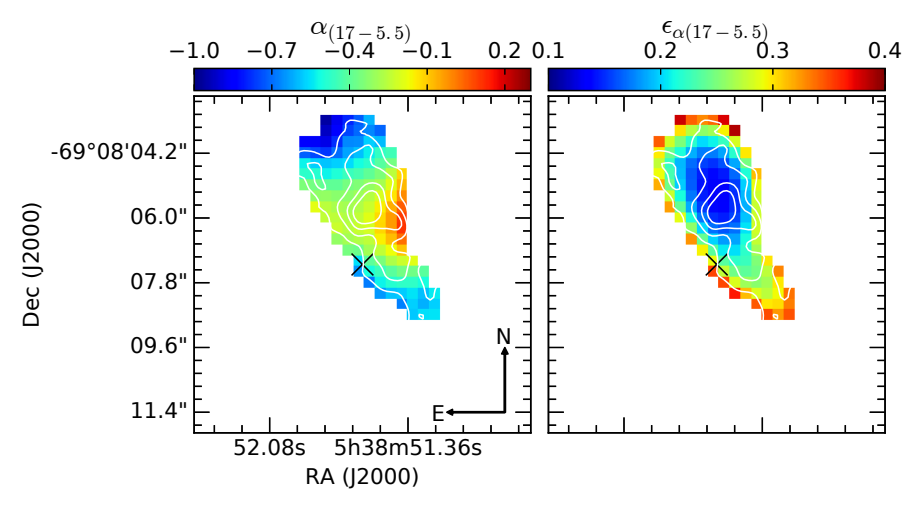

Fig. 8. Left: spectral index map between 17 and $5.5 \mathrm{GHz}$. Right: error spectral index map in Jy pixel ${ }^{-1}$. The map at $17 \mathrm{GHz}$ was reconvolved in order to match the beam at $5.5 \mathrm{GHz}$. The white contours indicate flux densities above $3,5,7,9 \sigma$ of the $17 \mathrm{GHz}$ emission. The cross indicates the position of the star.

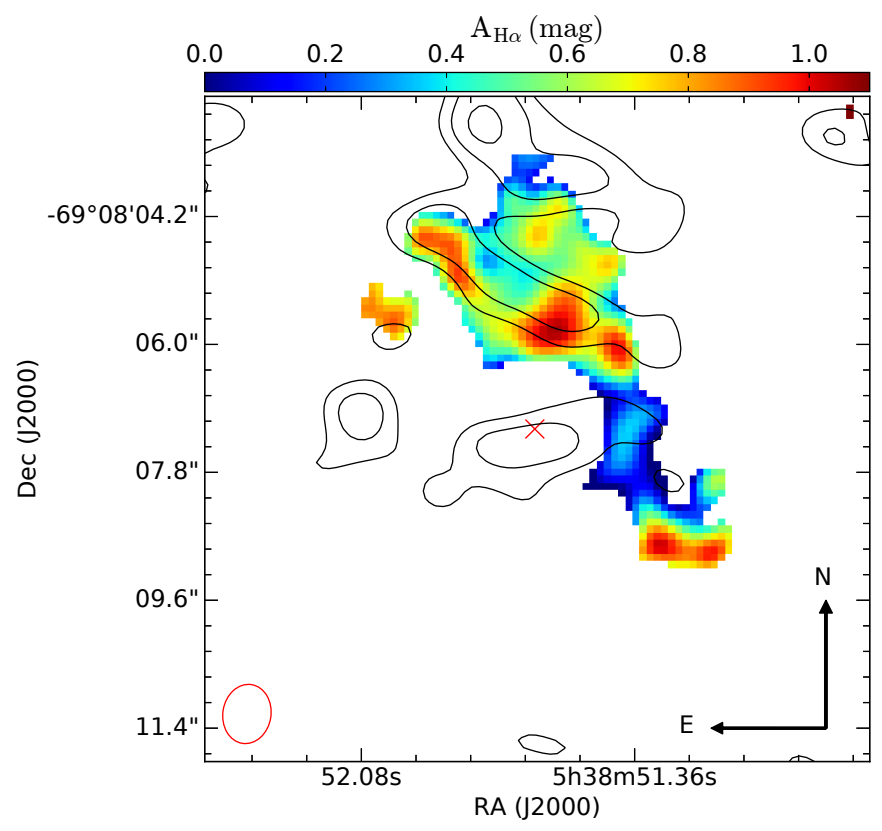

Fig. 9. Extinction map in $\mathrm{H} \alpha$ derived by comparing the $\mathrm{H} \alpha$ recombination line and the centimetre $(17 \mathrm{GHz})$ emission above $3 \sigma$. The central star, indicated with the red cross, is masked with a circular aperture in the optical image. The sub-mm contours at $343 \mathrm{GHz}$ are shown (black lines).

surface brightness at optical and radio wavelengths (Pottasch 1984).

We follow the procedure described in Agliozzo et al. (2017b). An electron temperature of $12200 \mathrm{~K}$ (Smith et al. 1998) is adopted. The extinction map is derived by comparing pixel-by-pixel the highest-resolution radio image $(17 \mathrm{GHz})$

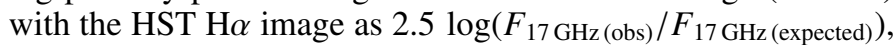

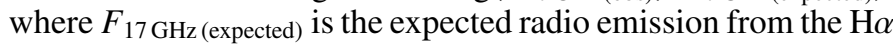
recombination-line emission (see Eqs. (7) and (8) in Agliozzo et al. 2017b). This computation was performed in every common pixel with brightness above $3 \sigma$, where $\sigma$ was computed by summing in quadrature the noise in the maps and calibration uncertainties. We mask the $\mathrm{H} \alpha$ emission from the star, keeping in mind that we want to estimate the expected free-free emission from the optical nebular line. The derived extinction map is illustrated in Fig. 9.
Relatively small extinction due to dust is evident across the entire region. The maximum value for $A_{\mathrm{H} \alpha}$ is $\sim 1.06$ mag and corresponds to the knot of the radio and optical emission. This knot is not a density clump according to the spectral index analysis and is likely the effect of material along the line of sight. The most extinguished region is in the same direction of the ALMA emission corresponding to the middle part of the nebula. The ALMA emission may arise mostly from a cool dust component that surrounds the ionized gas in the nebula and extinguishes the optical $\mathrm{H} \alpha$ emission.

\section{The SED of RMC 143}

Our ALMA sub-mm observations can constrain some of the dust properties. To understand the origin of the ALMA sub-mm emission we compare it with the available IR photometry, which were derived from images with different spatial resolutions. We consulted the IR catalogues with the CDS VizieR (Ochsenbein et al. 2000) and IRSA Gator ${ }^{4}$ tools. For the mid- and far-IR photometry we evaluated the effect of confusion on photometry. We include photometry from the 2MASS Point Source Catalog (Cutri et al. 2003), the Spitzer SAGE legacy survey (Meixner et al. 2006, Data Release 3/final), the AKARI IRC LMC survey (Kato et al. 2012; Ita et al. 2008), WISE (Cutri et al. 2012), and Herschel PACS (Meixner et al. 2013). We include our measurements of the ALMA Band 7 and ATCA Bands $4 \mathrm{~cm}(5.5$ and $9 \mathrm{GHz})$ and $15 \mathrm{~mm}(17 \mathrm{GHz})$.

We have excluded the WISE W3 and W4 band photometry due to confusion in the relatively large effective beams $\left(\sim 7^{\prime \prime}\right.$ for $\mathrm{W} 3$ and $\sim 12^{\prime \prime}$ for W4, $\sqrt{2}$ larger in Atlas images) and the complication of the very wide W3 spectral response. The W4 band photometry in particular is highly discrepant with the MIPS24 photometry at similar wavelength but much higher spatial resolution (5.9" FWHM). We consider as valid the AKARI S11 and L15 band photometry. Although the AKARI all-sky survey does not have sufficient spatial resolution, the LMC survey was taken in a pointed imaging mode which yields comparable resolution to MIPS24 in the L15 band and even better in the S11 band (FWHM of 5.7" for L15 and 4.8" for S11, Onaka et al. 2007). The available Herschel PACS100 and 160 band photometry is somewhat affected by confusion due to the large PSFs (7.7" and 12" FWHM), but this appears to be well accounted for in the large quoted error bars, and they are the only far-IR photometry available. Unfortunately, there is no PACS70 data for RMC 143, which would have had a better spatial resolution and been closer to the peak of the dust emission. There is no detection by MIPS70 due to the very large beam $\left(18^{\prime \prime}\right.$ FWHM) and confusion. For MIPS24 we take the weighted average of the two SAGE epoch measurements of $2.974 \pm 0.016 \mathrm{mag}$ and $2.913 \pm 0.010 \mathrm{mag}$, that is, $2.930 \mathrm{mag}$ or $482.5 \mathrm{mag}$. Due to the expected confusion we assume a $20 \%$ uncertainty on the MIPS24 photometry.

We also include the photometry available in VizieR from the UV and optical catalogues XMM-Newton, AC2000, AAVSO, Gaia, and RAVE (Page et al. 2012; Urban et al. 1998; Henden et al. 2015; Gaia Collaboration 2016; Kunder et al. 2017). We correct these data for interstellar dust extinction, using the extinction curve from Fitzpatrick (1999), $R_{V}=4.0$ and $E(B-V)=0.42 \mathrm{mag}$, as determined from our CMFGEN modelling (see Table 2). Keeping in mind that the UV to near-IR photometry comes from observations performed at different epochs and the star being likely variable, we can argue that

\footnotetext{
4 https://irsa.ipac.caltech.edu/applications/Gator/
} 


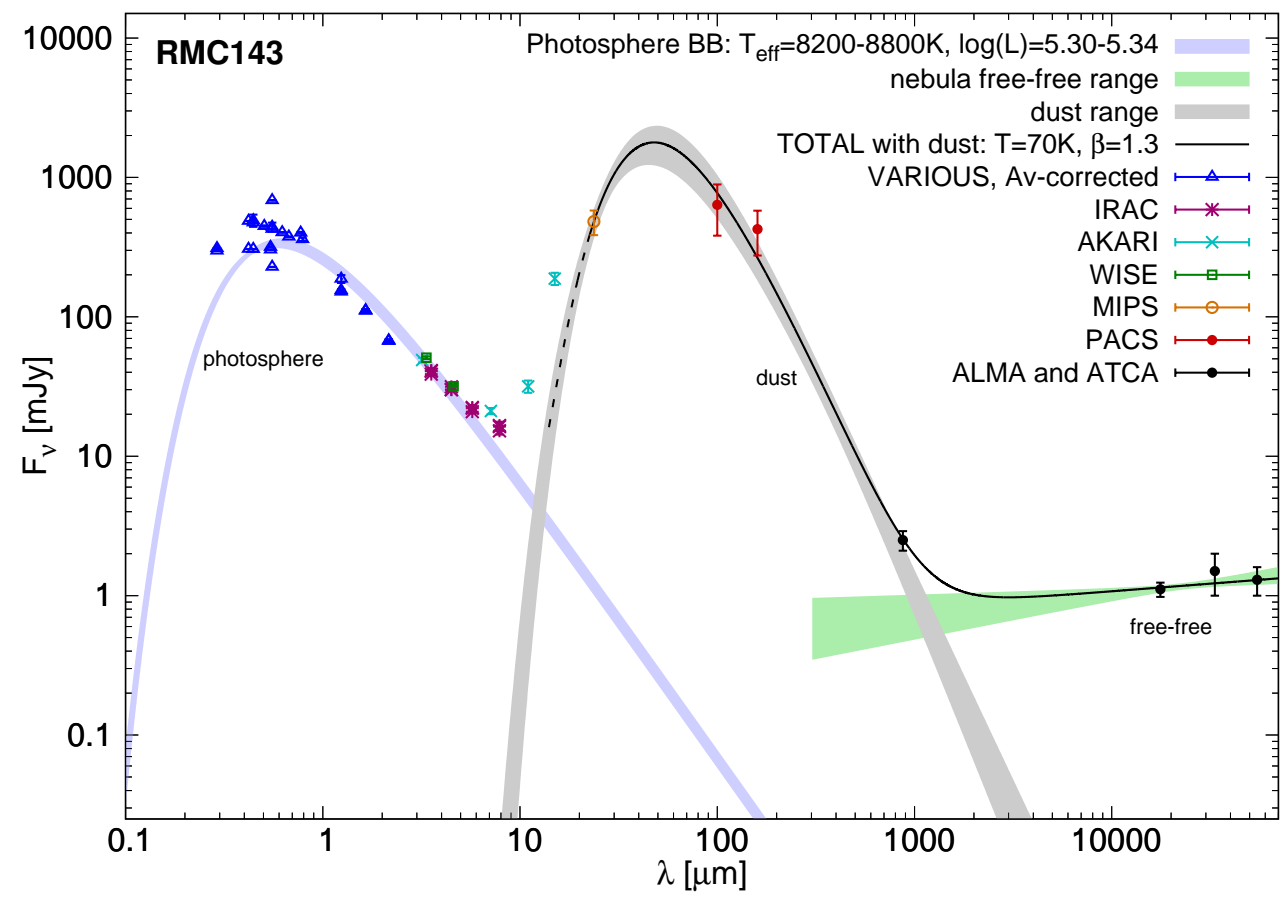

Fig. 10. Flux density distribution of RMC 143 from IR catalogues and our ALMA and ATCA measurements. The dust component shown is a greybody fit to the MIPS24, PACS100, PACS160, and ALMA photometry. The grey and green bands show the ranges of values within twice the minimum $\chi^{2}$ for the dust greybody and nebula free-free fits, respectively. We note that the AKARI S11 and L15 photometry have a colour correction applied assuming a $T=70 \mathrm{~K}$, $\beta=1$ greybody. the corrected data (blue triangles in Fig. 10) agree with the CMFGEN model of a star with $T_{\text {eff }}=8500 \pm 300 \mathrm{~K}$, $\log \left(L_{*} / L_{\odot}\right)=5.32$ at a distance of $49.97 \mathrm{kpc}$ (Table 2).

The SED of RMC 143's circumstellar material can be described with two components: (1) the ionized gas in the nebula that can be modelled by optically thin bremsstrahlung dominating the total flux at radio wavelengths (see Sect. 6); (2) a dust component that peaks in the far-IR, typical for LBV nebulae (e.g. Hutsemekers 1997; Clark et al. 2003).

Given the limitations of the available photometry and lack of a mid-IR spectrum, we fit only a simple single-temperature greybody (power-law opacity index $\beta$, see e.g. Backman \& Paresce 1993) to model the dust and obtain an estimate of the dust mass. As wavelengths shortward of about $20 \mu \mathrm{m}$ can deviate strongly from a simple greybody due to $\mathrm{PAH}$ and/or silicate spectral features (typically in HII regions, but also in the nebula of the candidate LBV HD 168625, Draine \& Li 2007; Compiègne et al. 2010; Umana et al. 2010) or due to several ionized gas forbidden lines (e.g. as seen in Spitzer IRS spectra of HR Car and G79.29+0.46; Umana et al. 2009; Agliozzo et al. 2014), we only fit the greybody to the MIPS24, PACS100+160, and ALMA photometry. Table 4 contains a summary of the photometry used to fit the dust. The best-fit photosphere and ionized gas free-free flux density distributions were added to the greybody for fitting the observed photometry. The free-free contribution to the ALMA measurement is significant at 0.47 to $1.01 \mathrm{mJy}$ (limits of twice minimum $\chi^{2}$ of the free-free fit to the ATCA photometry), assuming the free-free power-law spectrum continues without turnover to the ALMA frequency. The uncertainty on the freefree contribution of $\pm 0.27 \mathrm{mJy}$ was added in quadrature with the ALMA measurement error in the fit.

A dust emissivity at $850 \mu \mathrm{m}$ of $\kappa_{850}=1.7 \mathrm{~cm}^{2} \mathrm{~g}^{-1}$ and LMC distance of $49.97 \mathrm{kpc}$ are assumed. The greybody fit yields representative parameters of $T_{\text {dust }}=70.9 \pm 8.9 \mathrm{~K}, \beta=1.31 \pm 0.30$ and $M_{\text {dust }}=0.055 \pm 0.018 M_{\odot}$. The reduced $\chi^{2}$ of the fit is 1.44 . This somewhat high value appears to be mostly due to the PACS160 measurement, which we do indeed expect to be the most affected by confusion due to having the largest beam size. The greybody flux density distribution region within twice the minimum $\chi^{2}$ is shown as the grey band in Fig. 10. The total model, including best-fit free-free, dust greybody and photosphere is shown by the black line (solid longward of $20 \mu \mathrm{m}$ where we consider the greybody meaningful). The AKARI S11 and L15 points in Fig. 10 have a colour-correction applied appropriate for a $T=70 \mathrm{~K}$, $\beta=1$ greybody (correction factors are 2.712 and 2.135, respectively, AKARI IRC Data User Manual), but they still lie significantly above the photosphere and the greybody. As previously mentioned, we expect these bands, which have a wide bandwidth, to show evidence of silicate or PAH emission, like in the case of the Galactic candidate LBV HD 168625 (Skinner 1997; Umana et al. 2010), or several bright mid-IR ionized gas emission lines, like in the case of HR Car and G79.29+0.46 (Umana et al. 2009, 2011a; Agliozzo et al. 2014), together with a possible hotter thermal component. Another source of excess at the mid-IR wavelengths could be very small grains, typically observed in HII regions and produced by erosion of large grains in the diffuse medium. Contribution from very small grains to the emissivity in the far-IR and sub-mm should be negligible (Compiègne et al. 2010) and we are not aware of their observations in LBV nebulae.

Note the departure of $\beta$ from the interstellar case $(\beta=2)$ : $\beta \sim 1.3$ implies either the existence of relatively large grains, a significant optical depth, or a range of physical dust temperatures (studies of Galactic objects showed that the dust temperature decreases with increasing distance from the star, e.g. Hutsemekers 1997; Buemi et al. 2017). Large grains have been found in other LBV nebulae, such as those of AG Car (McGregor et al. 1988b; Vamvatira-Nakou et al. 2015) and $\eta$ Car (Mitchell \& Robinson 1986), and in the ejecta of RSGs (e.g. Scicluna et al. 2015). The derived temperatures are higher than typical values of interstellar dust and also dust in evolved HII regions (Paladini et al. 2012). The dust temperature is typical compared to other LBVs (e.g. Hutsemekers 1997). Stars displaying the $\mathrm{B}[\mathrm{e}]$ phenomenon have higher dust temperatures of about 500-1000 K (Lamers et al. 1998). A detailed modelling of the spatially integrated SED of $\eta$ Car's Homunculus including 
Table 4. Photometry used in the dust greybody fit.

\begin{tabular}{llccc}
\hline \hline Telescope & Instr. & $\begin{array}{c}\lambda \\
(\mu \mathrm{m})\end{array}$ & $\begin{array}{c}F W H M \\
\left({ }^{\prime \prime}\right)\end{array}$ & $\begin{array}{c}S_{v} \\
(\mathrm{mJy})\end{array}$ \\
\hline Spitzer & MIPS & 23.675 & 5.9 & $482.5 \pm 96.5^{(a)}$ \\
Herschel & PACS & 100 & 6.7 & $636 \pm 254$ \\
Herschel & PACS & 160 & 11 & $426 \pm 151$ \\
ALMA & Band 7 & 872.8 & $1.1^{(b)}$ & $2.5 \pm 0.4$ \\
\hline
\end{tabular}

Notes. ${ }^{(a)}$ MIPS24 uncertainty set to $20 \%$ to account for confusion.

${ }^{(b)}$ ALMA measurement is integrated over the nebula.

Table 5. Summary of available LMC LBVs dust properties.

\begin{tabular}{lcccr}
\hline \hline Source & $\begin{array}{c}\text { Dust mass } \\
\left(10^{-2} M_{\odot}\right)\end{array}$ & $\begin{array}{c}\text { Dust T } \\
(\mathrm{K})\end{array}$ & $\beta$ & Ref. \\
\hline RMC 143 & $5.5 \pm 1.8$ & $62-80$ & $1.0-1.6$ & This work \\
RMC 127 & $0.2-2$ & $71-90$ & $1.5-2.0$ & {$[1]$} \\
S 61 & $0.5-3$ & $105-145$ & $0.55-1.5$ & {$[2]$} \\
RMC 71 & 1 & N/A & N/A & {$[3]$} \\
\hline
\end{tabular}

References. [1] Agliozzo et al. (2017a); [2] Agliozzo et al. (2017b); [3] Guha Niyogi et al. (2014).

mid- and far-IR spectroscopy by Morris et al. (2017) shows a very complex chemical composition of the dusty ejecta. Unfortunately, the current data of RMC 143 preclude us to derive a more detailed model. Note that RMC 143 has a significant fraction of ionized gas, while the Homunculus is mostly neutral, so the mid-IR spectrum might be different in the two objects.

\section{The nebular mass and initial mass}

The sub-mm emission detected with ALMA presents a different morphology than the optical and radio emission, although the contours have low statistical significance due to the low signalto-noise ratio. The sub-mm emission on the north-east side of the nebula may be aligned with the interstellar filaments associated with 30 Dor. An analysis of the data shows that: (1) at least two-thirds of the integrated sub-mm flux density is due to dust that extinguishes the $\mathrm{H} \alpha$ emission in-homogeneously across the nebula; (2) dust temperatures and parameter $\beta$ values are consistent with processed material in LBV nebulae rather than with ISM dust.

The dust mass derived in the previous section is to date the largest value found in an LBV nebula at sub-solar metallicities. Only a few studies on dust in the MCs LBVs have been performed, see Table 5. A study to address the contribution of LBVs to dust production at the LMC and SMC metallicities is ongoing (Agliozzo et al., in prep.).

The gas-to-dust ratio G/D is an unknown parameter in LBVs. Because of favourable physical conditions in LBV eruptions, this parameter may be lower than in the ISM. Roman-Duval et al. (2014) report a G/D of $\sim 400$ in the LMC diffuse atomic ISM, although they find that in dense regions this might be lower (G/D 200). Assuming the lower value as representative of the 30 Dor region and the dust mass derived in this work, we find a total nebular mass $M_{\mathrm{TOT}}$ for RMC 143 of $11.0 \pm 3.7 M_{\odot}$, and assuming $\mathrm{G} / \mathrm{D}=100$ (more representative of our Galaxy) $M_{\mathrm{TOT}}=5.5 \pm 1.8 M_{\odot}$.

In Agliozzo et al. (2012), we derived an approximate ionized mass of $\sim 0.5-0.9 M_{\odot}$. Although the ionized mass estimate is dependent on the assumed volume for the nebula (which is not easy to determine because of the nebula's irregular shape), it seems plausible that a large gas mass is not ionized and is located in a photo-dissociation region around the ionized nebula, mixed with the dust. The total nebular mass has an order of magnitude uncertainty, due mostly to the uncertainty of the gas to dust ratio and of the assumed dust emissivity, $\kappa_{850}$.

The stellar parameters of RMC 143 suggest that the star's location in the HR diagram is at the lower luminosity end of LBVs. Our derived luminosity $\left(\log \left(L / L_{\odot}\right)=5.32\right)$ suggests a single-star initial mass around $25-30 M_{\odot}$. Given that the CMFGEN models suggest a (spectroscopic) current mass of $\sim 8 M_{\odot}$, it appears that RMC 143 has lost a large fraction of its initial mass. RMC 143's position in the HR diagram is similar to that of the Yellow Hypergiant (YHG) Hen3-1379, a post-Red Supergiant (RSG) star on its way to the LBV phase, as argued by Lagadec et al. (2011) and Hutsemékers et al. (2013). Further analysis would be needed to draw firm conclusions on whether the nebula was ejected during binary interaction, or LBV eruptions, or during a previous RSG phase. In particular, nebular abundances would need to be compared to numerical stellar evolution models of single and binary stars. Beasor \& Davies (2018) discussed the uncertainties of RSG mass-loss rates along the evolutionary sequence of a $16 M_{\odot}$ star, showing that such a RSG star loses a small fraction $\left(\sim 0.6 M_{\odot}\right)$ of its mass through stellar winds. However, no similar observational estimate exists for the more massive RSGs that RMC 143 could have evolved from.

In light of our revised low luminosity of RMC 143, an interesting avenue for future study would be a re-appraisal of the evolutionary status of RMC 143 in comparison to its nearby environment (Smith \& Tombleson 2015; Humphreys et al. 2016; Aadland et al. 2018). Aghakhanloo et al. (2017) developed models of passive dissolution of young stellar clusters, and concluded that LBV environments are inconsistent with them evolving from single stars. It would be interesting to investigate the behaviour of RMC 143 given its relatively low current and initial mass that we derived in this paper.

\section{The ionizing source of RMC 143's nebula}

The quiescent temperature of RMC 143 over the past $\sim 60 \mathrm{yr}$ suggests that the star is not hot enough to ionize its extended nebula. Although we cannot exclude a hotter phase for RMC 143 in the past, the observations suggest that an external ionizing source may be responsible for the ionization of its nebula. This was also proposed for the circumstellar nebulae of the Pistol Star (Figer et al. 1999) and IRAS 18576+0341 (Buemi et al. 2010).

The nearby star-forming region 30 Dor contains $>700$ massive stars (Doran et al. 2013), with a large fraction of them forming the well-known RMC 136 cluster. 30 Dor's giant nebula is ionized and detected at radio wavelengths (Fig. 5). Melnick (1985) conclude that a large fraction of the ionization of 30 Dor is provided by the UV photon budget produced by the early-type stars, WR stars, and blue supergiants within $25 \mathrm{pc}$ of the cluster centre. Doran et al. (2013) estimate the feedback of hot luminous stars in 30 Dor from a census of hot stars within $150 \mathrm{pc}$ of RMC 136 obtained by the VLT-FLAMES Tarantula Survey (Evans et al. 2011).

We use the ionizing photon luminosities in Doran et al. to derive an order of magnitude estimate of the Strömgren radius at different distances from RMC 136. In particular, we use the cumulative UV photon fluxes for the sample that includes the Grand Total of stars with spectroscopic classification from their Table 9. We derive the Strömgren radius as $R_{\mathrm{S}}=\left(3 \times S_{\mathrm{UV}} /\left(4 \pi n_{\mathrm{e}}^{2} \beta_{2}\right)\right)^{1 / 3}$, 
where $\beta_{2}=3 \times 10^{-13} \mathrm{~cm}^{3} \mathrm{~s}^{-1}$ (for typical ISM electron temperatures of $\sim 10000 \mathrm{~K}$ ) and $S_{\mathrm{UV}}$ is the UV photon flux per second, in the simplifying assumption that the gas is mostly hydrogen.

Accounting for only the stars within $5 \mathrm{pc}$ from the cluster centre, the integrated UV photon flux in Doran et al. is $576 \times 10^{49} \mathrm{ph} \mathrm{s}^{-1}$. The Strömgren radius decreases with the plasma density $n_{\mathrm{e}}$ as $n_{\mathrm{e}}^{-2 / 3}$. Thus, for two orders of magnitudes of plasma densities in the ISM and in star-forming regions, $30 \mathrm{~cm}^{-3}$ and $300 \mathrm{~cm}^{-3}, R_{\mathrm{S}}$ varies between 56 and $12 \mathrm{pc}$, respectively. The UV photon flux in the 30 Dor region doubles if stars at larger distances from the cluster centre are taken into account (up to a projected distance of $150 \mathrm{pc}$ ). For a total of $1056 \times 10^{49} \mathrm{ph} \mathrm{s}^{-1}$, $R_{\mathrm{S}}$ varies from about $15 \mathrm{pc}$ (for densities of $300 \mathrm{~cm}^{-3}$ ) to $68 \mathrm{pc}$ (for densities of $30 \mathrm{~cm}^{-3}$ ). RMC 143 has a projected distance of about 32 pc from RMC 136. Although we are considering projected distances, it seems plausible that RMC 143's nebula is ionized by an external ionizing source.

In addition, Doran et al. (2013) estimate that about $6 \%$ of the ionizing photons escape the region, although with large uncertainty. This implies that all hydrogen inside the region is in ionization equilibrium, including plausibly RMC 143's circumstellar nebula. A similar scenario ("inverted" photoionization; Schuster et al. 2009) was outlined to explain the asymmetric HII region associated with the Red Supergiant NML Cyg (Habing et al. 1982; Morris \& Jura 1983; Schuster et al. 2006). This phenomenon is also found in the YHGs and RSGs of the Westerlund 1 cluster in our Galaxy (Dougherty et al. 2010; Fenech et al. 2018; Andrews et al. 2018).

\section{Summary and conclusions}

We report the discovery of a massive dusty component in the nebula around LBV RMC 143, through resolved sub-mm observations performed with ALMA. The dust mass inferred from the available space telescope IR photometry and from the ALMA Band 7 observations is $0.055 \pm 0.018 M_{\odot}$ assuming $\kappa_{850}=1.7 \mathrm{~cm}^{2} \mathrm{~g}^{-1}$, a distance of $49.97 \mathrm{kpc}$, and an average dust temperature of $\sim 70 \mathrm{~K}$. The representative dust temperature is derived from a modified grey-body that best fits the MIPS24, PACS100, PACS160, and ALMA $850 \mu$ m photometry (integrated over the entire nebula). An additional mid-IR excess is seen in the broad-band AKARI S11 and L15 photometry, which could be due to a combination of emission lines from the ionized gas, silicate, and PAH emission bands.

We compare the ALMA dust detection with the $8 \mu \mathrm{m}$ Spitzer and the $\mathrm{H} \alpha$ HST images, and with the 5.5, 9, and $17 \mathrm{GHz}$ ATCA maps. The morphological comparison and the extinction map suggest that the dust is distributed in a photo-dissociation region partially surrounding the ionized nebula.

The historical light curve of RMC 143 and the past literature show that the previous LBV classification was likely the result of a mis-identification with another star. However, despite the possible lack of evidence of S Doradus cycles, the massive circumstellar nebula and the stellar parameters support the LBV classification. RMC 143 is at the lower luminosity end of LBVS $\left(\log \left(L / L_{\odot}\right)=5.32\right)$, with an effective temperature in 2015 of $\sim 8500 \mathrm{~K}$. The star may be in outburst phase for the last three decades, which would also explain the relatively high mass-loss rate of $1.0 \times 10^{-5} M_{\odot} \mathrm{yr}^{-1}$. The stellar wind is enhanced in $\mathrm{He}$ and $\mathrm{N}$, and depleted in $\mathrm{H}, \mathrm{C}$, and $\mathrm{O}$, confirming the evolved nature of the star. RMC 143 is currently not hot enough to ionize its circumstellar nebula. While the star may have been hotter in the past, the nebula could be kept in ionization equilibrium by the UV photons escaping the 30 Dor star-forming region.
The current stellar mass in our best-fit CMFGEN model is $\sim 8 M_{\odot}$, which is very low compared to the total nebular mass of $\sim 5.5 M_{\odot}$, derived assuming a gas-to-dust ratio of 100 and a dust mass value from the best-fit of the mid-IR to sub-mm data. This suggests that the star has already lost a large fraction of its initial mass, through past LBV eruptions or possibly binary interactions.

Because of its brightness across the electromagnetic spectrum, RMC 143 is ideal for detailed studies of its chemical and dust composition and kinematics of its circumstellar nebula. Future integral field unit spectroscopy and continuum highresolution observations (e.g. with Gemini/GMOS-S, VLT/Muse, ALMA, ELT and the James Webb Space Telescope) will shed light on the formation of RMC 143's massive circumstellar nebula.

Acknowledgements. We thank the reviewer, Simon Clark, for their careful review of the manuscript and their constructive remarks. We wish to thank the staff at ALMA, ATCA and ESO who made these observations possible. We also thank Jorge Melnick for trying to find the photographic plates of his observations of RMC 143. CA acknowledges support from FONDECYT grant No. 3150463. Support to CA and GP was provided by the Ministry of Economy, Development, and Tourism's Millennium Science Initiative through grant IC120009, awarded to The Millennium Institute of Astrophysics, MAS. This paper makes use of the following ALMA data: ADS/JAO.ALMA\#2013.1.00450.S. ALMA is a partnership of ESO (representing its member states), NSF (USA) and NINS (Japan), together with NRC (Canada) and NSC and ASIAA (Taiwan) and KASI (Republic of Korea), in cooperation with the Republic of Chile. The Joint ALMA Observatory is operated by ESO, AUI/NRAO and NAOJ. This paper also includes data collected: at the European Organisation for Astronomical Research in the Southern Hemisphere under ESO programme 096.D-0047(A); at the Australia Telescope Compact Array, which is part of the Australia Telescope National Facility which is funded by the Australian Government for operation as a National Facility managed by CSIRO. This work made use of PyAstronomy. This research has made use of the: International Variable Star Index (VSX) database, operated at AAVSO, Cambridge, Massachusetts, USA; VizieR catalogue access tool, CDS, Strasbourg, France. The original description of the VizieR service was published in A\&AS 143, 23.

\section{References}

Aadland, E., Massey, P., Neugent, K. F., \& Drout, M. R. 2018, AJ, 156, 294 Aghakhanloo, M., Murphy, J. W., Smith, N., \& Hložek, R. 2017, MNRAS, 472, 591

Agliozzo, C., Umana, G., Trigilio, C., et al. 2012, MNRAS, 426, 181

Agliozzo, C., Noriega-Crespo, A., Umana, G., et al. 2014, MNRAS, 440, 1391 Agliozzo, C., Trigilio, C., Pignata, G., et al. 2017a, ApJ, 841, 130

Agliozzo, C., Nikutta, R., Pignata, G., et al. 2017b, MNRAS, 466, 213

Andrews, H., Fenech, D., Prinja, R. K., Clark, J. S., \& Hindson, L. 2018, MNRAS, 477, L55

Backman, D. E., \& Paresce, F. 1993, in Protostars and Planets III, eds. E. H. Levy, \& J. I. Lunine, 1253

Beasor, E. R., \& Davies, B. 2018, MNRAS, 475, 55

Boffin, H. M. J., Rivinius, T., Mérand, A., et al. 2016, A\&A, 593, A90

Boian, I., \& Groh, J. H. 2018, A\&A, 617, A115

Buemi, C. S., Umana, G., Trigilio, C., Leto, P., \& Hora, J. L. 2010, ApJ, 721, 1404

Buemi, C. S., Trigilio, C., Leto, P., et al. 2017, MNRAS, 465, 4147

Cannon, A. J. 1936, Annals of Harvard College Observatory, 100, 205

Clark, J. S., Egan, M. P., Crowther, P. A., et al. 2003, A\&A, 412, 185

Clark, J. S., Larionov, V. M., \& Arkharov, A. 2005, A\&A, 435, 239

Clark, J. S., Crowther, P. A., Larionov, V. M., et al. 2009, A\&A, 507, 1555

Clark, J. S., Castro, N., Garcia, M., et al. 2012, A\&A, 541, A146

Clayton, G. C., \& Martin, P. G. 1985, ApJ, 288, 558

Compiègne, M., Flagey, N., Noriega-Crespo, A., et al. 2010, ApJ, 724, L44 Conti, P. S. 1984, in Observational Tests of the Stellar Evolution Theory, eds. A. Maeder, \& A. Renzini, IAU Symp., 105, 233

Conti, P. S. 1997, in Luminous Blue Variables: Massive Stars in Transition, eds. A. Nota, \& H. Lamers, ASP Conf. Ser., 120, 387

Crowther, P. A. 2007, ARA\&A, 45, 177

Cutri, R. M., Skrutskie, M. F., van Dyk, S., et al. 2003, VizieR Online Data Catalog: II/246 
Cutri, R. M., Skrutskie, M. F., van Dyk, S., et al. 2012, VizieR Online Data Catalog: II/281

Davidson, K., \& Humphreys, R. M. 1997, ARA\&A, 35, 1

Davidson, K., \& Humphreys, R. M. 2012, in Eta Carinae and the Supernova Impostors, Astrophys. Space Sci. Lib., 384

de Groot, M. 1988, Ir. Astron. J., 18, 163

Doran, E. I., Crowther, P. A., de Koter, A., et al. 2013, A\&A, 558, A134

Dougherty, S. M., Clark, J. S., Negueruela, I., Johnson, T., \& Chapman, J. M. 2010, A\&A, 511, A58

Draine, B. T., \& Li, A. 2007, ApJ, 657, 810

Egan, M. P., Clark, J. S., Mizuno, D. R., et al. 2002, ApJ, 572, 288

Evans, C. J., Taylor, W. D., Hénault-Brunet, V., et al. 2011, A\&A, 530, A108

Feast, M. W. 1961, MNRAS, 122, 1

Fenech, D. M., Clark, J. S., Prinja, R. K., et al. 2018, A\&A, 617, A137

Figer, D. F., Morris, M., Geballe, T. R., et al. 1999, ApJ, 525, 759

Fitzpatrick, E. L. 1999, PASP, 111, 63

Flagey, N., Noriega-Crespo, A., Petric, A., \& Geballe, T. R. 2014, AJ, 148, 34

Gaia Collaboration (Prusti, T., et al.) 2016, A\&A, 595, A1

Gal-Yam, A., \& Leonard, D. C. 2009, Nature, 458, 865

Gallagher, J. S. 1989, in IAU Colloq. 113: Physics of Luminous Blue Variables, eds. K. Davidson, A. F. J. Moffat, \& H. J. G. L. M. Lamers, Astrophys. Space Sci. Lib., 157, 185

Georgiev, L., Koenigsberger, G., Hillier, D. J., et al. 2011, AJ, 142, 191

Groh, J. H. 2011, J. Phys. Conf. Ser., 328, 012020

Groh, J. H. 2014, A\&A, 572, L11

Groh, J. H., \& Vink, J. S. 2011, A\&A, 531, L10

Groh, J. H., Hillier, D. J., \& Damineli, A. 2006, ApJ, 638, L33

Groh, J. H., Hillier, D. J., Damineli, A., et al. 2009, ApJ, 698, 1698

Groh, J. H., Hillier, D. J., \& Damineli, A. 2011, ApJ, 736, 46

Groh, J. H., Meynet, G., \& Ekström, S. 2013, A\&A, 550, L7

Groh, J. H., Meynet, G., Ekström, S., \& Georgy, C. 2014, A\&A, 564, A30

Guha Niyogi, S., Min, M., Meixner, M., et al. 2014, A\&A, 569, A80

Guzik, J. A., \& Lovekin, C. C. 2014, ArXiv e-prints [arXiv:1402 . 0257]

Gvaramadze, V. V., Kniazev, A. Y., \& Fabrika, S. 2010, MNRAS, 405, 1047

Habing, H. J., Goss, W. M., \& Winnberg, A. 1982, A\&A, 108, 412

Henden, A. A., Levine, S., Terrell, D., \& Welch, D. L. 2015, Amer. Astron. Soc. Meet. Abstr., \#225, 225, 336.16

Hillier, D. J., \& Miller, D. L. 1998, ApJ, 496, 407

Hillier, D. J., Davidson, K., Ishibashi, K., \& Gull, T. 2001, ApJ, 553, 837

Humphreys, R. M., \& Davidson, K. 1994, PASP, 106, 1025

Humphreys, R. M., Weis, K., Davidson, K., \& Gordon, M. S. 2016, ApJ, 825, 64

Humphreys, R. M., Gordon, M. S., Martin, J. C., Weis, K., \& Hahn, D. 2017, ApJ, 836, 64

Hutsemekers, D. 1997, in Luminous Blue Variables: Massive Stars in Transition, eds. A. Nota, \& H. Lamers, ASP Conf. Ser., 120, 316

Hutsemekers, D., \& van Drom, E. 1991, A\&A, 248, 141

Hutsemékers, D., Cox, N. L. J., \& Vamvatira-Nakou, C. 2013, A\&A, 552, L6

Ita, Y., Onaka, T., Kato, D., et al. 2008, PASJ, 60, S435

Jayasinghe, T., Kochanek, C. S., Stanek, K. Z., et al. 2018, MNRAS, 477, 3145

Justham, S., Podsiadlowski, P., \& Vink, J. S. 2014, ApJ, 796, 121

Kashi, A. 2010, in AIP Conf. Ser., eds. V. Kalogera, \& M. van der Sluys, 1314 55

Kashi, A., \& Soker, N. 2010, ApJ, 723, 602

Kato, D., Ita, Y., Onaka, T., et al. 2012, AJ, 144, 179

Kochanek, C. S. 2011, ApJ, 743, 73

Kochanek, C. S., Shappee, B. J., Stanek, K. Z., et al. 2017, PASP, 129, 104502

Kotak, R., \& Vink, J. S. 2006, A\&A, 460, L5

Kunder, A., Kordopatis, G., Steinmetz, M., et al. 2017, AJ, 153, 75

Lagadec, E., Zijlstra, A. A., Oudmaijer, R. D., et al. 2011, A\&A, 534, L10

Lamers, H. J. G. L. M., \& de Groot, M. J. H. 1992, A\&A, 257, 153

Lamers, H. J. G. L. M., Zickgraf, F.-J., de Winter, D., Houziaux, L., \& Zorec, J. 1998, A\&A, 340, 117

Lang, C. C., Figer, D. F., Goss, W. M., \& Morris, M. 1999, AJ, 118, 2327

Langer, N., Hamann, W.-R., Lennon, M., et al. 1994, A\&A, 290, 819

Lau, R. M., Herter, T. L., Morris, M. R., \& Adams, J. D. 2014, ApJ, 785, 120

Leitherer, C., Chapman, J. M., \& Koribalski, B. 1995, ApJ, 450, 289

Makovoz, D., \& Marleau, F. R. 2005, PASP, 117, 1113

Martins, F. 2011, Bull. Soc. Roy. Sci. Liege, 80, 29

McGregor, P. J., Hillier, D. J., \& Hyland, A. R. 1988a, ApJ, 334, 639
McGregor, P. J., Finlayson, K., Hyland, A. R., et al. 1988b, ApJ, 329, 874 Mehner, A., Baade, D., Groh, J. H., et al. 2017, A\&A, 608, A124 Meixner, M., Gordon, K. D., Indebetouw, R., et al. 2006, AJ, 132, 2268 Meixner, M., Panuzzo, P., Roman-Duval, J., et al. 2013, AJ, 146, 62

Melnick, J. 1985, A\&A, 153, 235

Mitchell, R. M., \& Robinson, G. 1986, MNRAS, 222, 347

Morris, M., \& Jura, M. 1983, ApJ, 267, 179

Morris, P. W., Gull, T. R., Hillier, D. J., et al. 2017, ApJ, 842, 79

Morse, J. A., Davidson, K., Bally, J., et al. 1998, AJ, 116, 2443

NASA/IPAC Infrared Science Archive, JPL, Caltech, \& NASA 2011, Astrophysics Source Code Library [record ascl:1111.006]

Nota, A., \& Lamers, H. 1997, in Luminous Blue Variables: Massive Stars in Transition, ASP Conf. Ser., 120

Nota, A., Livio, M., Clampin, M., \& Schulte-Ladbeck, R. 1995, ApJ, 448, 788

Nowak, M., Flagey, N., Noriega-Crespo, A., et al. 2014, ApJ, 796, 116

Ochsenbein, F., Bauer, P., \& Marcout, J. 2000, A\&AS, 143, 23

Onaka, T., Matsuhara, H., Wada, T., et al. 2007, PASJ, 59, S401

Owocki, S. P. 2015, in Very Massive Stars in the Local Universe, ed. J. S. Vink, Astrophys. Space Sci. Lib., 412, 113

Page, M. J., Brindle, C., Talavera, A., et al. 2012, MNRAS, 426, 903

Paladini, R., Umana, G., Veneziani, M., et al. 2012, ApJ, 760, 149

Panagia, N., \& Felli, M. 1975, A\&A, 39, 1

Parker, J. W., Clayton, G. C., Winge, C., \& Conti, P. S. 1993, ApJ, 409, 770

Paron, S., Combi, J. A., Petriella, A., \& Giacani, E. 2012, A\&A, 543, A23

Pojmanski, G. 1997, Acta Astron., 47, 467

Portegies Zwart, S. F., \& van den Heuvel, E. P. J. 2016, MNRAS, 456, 3401

Pottasch, S. R. 1984, S\&T, 67, 527

Richardson, N. D., \& Mehner, A. 2018, RNAAS, 2, 121

Ritchie, B. W., Clark, J. S., Negueruela, I., \& Najarro, F. 2009, A\&A, 507, 1597

Rizzo, J. R., Jiménez-Esteban, F. M., \& Ortiz, E. 2008, ApJ, 681, 355

Rizzo, J. R., Palau, A., Jiménez-Esteban, F., \& Henkel, C. 2014, A\&A, 564, A21

Roman-Duval, J., Gordon, K. D., Meixner, M., et al. 2014, ApJ, 797, 86

Schuster, M. T., Humphreys, R. M., \& Marengo, M. 2006, AJ, 131, 603

Schuster, M. T., Marengo, M., Hora, J. L., et al. 2009, ApJ, 699, 1423

Scicluna, P., Siebenmorgen, R., Wesson, R., et al. 2015, A\&A, 584, L10

Skinner, C. J. 1997, in Luminous Blue Variables: Massive Stars in Transition, eds. A. Nota, \& H. Lamers, ASP Conf. Ser., 120, 322

Smith, N. 2011, MNRAS, 415, 2020

Smith, N. 2013, MNRAS, 429, 2366

Smith, N., \& Frew, D. J. 2011, MNRAS, 415, 2009

Smith, N., \& Tombleson, R. 2015, MNRAS, 447, 598

Smith, L. J., Nota, A., Pasquali, A., et al. 1998, ApJ, 503, 278

Smith, N., Gehrz, R. D., Hinz, P. M., et al. 2003, AJ, 125, 1458

Smith, N., Li, W., Foley, R. J., et al. 2007, ApJ, 666, 1116

Smith, N., Chornock, R., Li, W., et al. 2008, ApJ, 686, 467

Thackeray, A. D. 1950, MNRAS, 110, 343

Trundle, C., Kotak, R., Vink, J. S., \& Meikle, W. P. S. 2008, A\&A, 483, L47

Tuthill, P. G., Monnier, J. D., Lawrance, N., et al. 2008, ApJ, 675, 698

Ueta, T., Meixner, M., Dayal, A., et al. 2001, ApJ, 548, 1020

Umana, G., Buemi, C. S., Trigilio, C., \& Leto, P. 2005, A\&A, 437, L1

Umana, G., Buemi, C. S., Trigilio, C., et al. 2009, ApJ, 694, 697

Umana, G., Buemi, C. S., Trigilio, C., Leto, P., \& Hora, J. L. 2010, ApJ, 718, 1036

Umana, G., Buemi, C. S., Trigilio, C., et al. 2011a, ApJ, 739, L11

Umana, G., Buemi, C. S., Trigilio, C., et al. 2011b, Bull. Soc. Roy. Sci. Liege, 80,335

Umana, G., Ingallinera, A., Trigilio, C., et al. 2012, MNRAS, 427, 2975

Urban, S. E., Corbin, T. E., Wycoff, G. L., et al. 1998, AJ, 115, 1212

Vamvatira-Nakou, C., Hutsemékers, D., Royer, P., et al. 2013, A\&A, 557, A20

Vamvatira-Nakou, C., Hutsemékers, D., Royer, P., et al. 2015, A\&A, 578, A108

van Genderen, A. M. 2001, A\&A, 366, 508

Vernet, J., Dekker, H., D’Odorico, S., et al. 2011, A\&A, 536, A105

Vink, J. S., \& de Koter, A. 2002, A\&A, 393, 543

Wachter, S., Mauerhan, J., van Dyk, S., Hoard, D. W., \& Morris, P. 2011, Bull.

Soc. Roy. Sci. Liege, 80, 291

Walborn, N. R., \& Blades, J. C. 1997, ApJS, 112, 457

Walborn, N. R., Gamen, R. C., Morrell, N. I., et al. 2017, AJ, 154, 15

Weis, K. 2003, A\&A, 408, 205

Wolf, B. 1989, A\&A, 217, 87 